Geometry $\&$ Topology

Volume 8 (2004) 611-644

Published: 12 April 2004

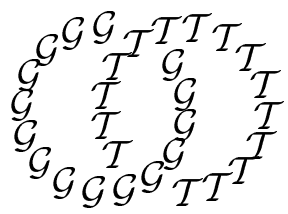

\title{
Finiteness properties of soluble arithmetic groups over global function fields
}

\author{
KAI-Uwe Bux \\ Cornell University, Department of Mathemtics \\ Malott Hall 310, Ithaca, NY 14853-4201, USA \\ Email: bux_math_2004@kubux.net \\ URL: http://www.kubux.net
}

\begin{abstract}
Let $\mathcal{G}$ be a Chevalley group scheme and $\mathcal{B} \leq \mathcal{G}$ a Borel subgroup scheme, both defined over $\mathbb{Z}$. Let $K$ be a global function field, $S$ be a finite non-empty set of places over $K$, and $\mathcal{O}_{S}$ be the corresponding $S$-arithmetic ring. Then, the $S$ arithmetic group $\mathcal{B}\left(\mathcal{O}_{S}\right)$ is of type $\mathrm{F}_{|S|-1}$ but not of type $\mathrm{FP}_{|S|}$. Moreover one can derive lower and upper bounds for the geometric invariants $\Sigma^{m}\left(\mathcal{B}\left(\mathcal{O}_{S}\right)\right)$. These are sharp if $\mathcal{G}$ has rank 1 . For higher ranks, the estimates imply that normal subgroups of $\mathcal{B}\left(\mathcal{O}_{S}\right)$ with abelian quotients, generically, satisfy strong finiteness conditions.
\end{abstract}

\section{AMS Classification numbers Primary: 20G30}

Secondary: $20 \mathrm{~F} 65$

Keywords: Arithmetic groups, soluble groups, finiteness properties, actions on buildings

Proposed: Benson Farb

Seconded: Martin Bridson, Steven Ferry
Received: 10 April 2003

Revised: 8 April 2004 
Once upon a time, all finitely generated groups were finitely presented. There were discontinuous subgroups of Lie groups or groups acting nicely on beautiful geometries - one should think, for example, of finitely generated Fuchsian groups. Then B H Neumann gave the first example of a finitely generated but infinitely related group in [33] where he even showed that there are uncountably many 2 generator groups. Among these, of course, only countably many are finitely presented. Hence finite presentability is a much stronger property than finite generation.

More than twenty years later, working on decision problems, G Baumslag, W W Boone, and BH Neumann showed that even finitely generated subgroups of finitely presented groups need not be finitely presented [7].

However, it took almost another twenty years until finitely generated infinitely related groups were "observed in nature". In [39, U Stuhler proved that the groups $\mathrm{SL}_{2}\left(k\left[t, t^{-1}\right]\right)$, where $k$ is a finite field, are finitely generated but not finitely presented. He extended these results in [40] constructing series of groups with increasing finiteness properties. In [38, $\mathrm{R}$ Strebel gives a historical and systematic survey with focus on soluble groups.

Because of the topological background of finite generation and finite presentability, there are two generalizations to higher dimensions: one based on homotopy groups, the other based on homology. A group $G$ is of type $F_{m}$ if there is an Eilenberg-MacLane space $K(G, 1)$ with finite $m$-skeleton. $G$ is of type $F P_{m}$ if the trivial $\mathbb{Z} G$-module $\mathbb{Z}$ admits a projective resolution that is finitely generated in dimensions $\leq m$. This is a homological variant of the homotopical finiteness property $\mathrm{F}_{m}$. The definition of type $\mathrm{F}_{m}$ was given by $\mathrm{CTC}$ Wall in 43. It is convenient to define the finiteness length of a group to be the largest dimension $m$ for which a group is of type $\mathrm{F}_{m}$.

A group is of type $\mathrm{F}_{1}$ if and only if it is finitely generated. Moreover, type $\mathrm{F}_{1}$ and type $\mathrm{FP}_{1}$ are equivalent notions. A group is of type $\mathrm{F}_{2}$ if and only if it is finitely presented. M Bestvina and N Brady [11] have given an example of a group of type $\mathrm{FP}_{2}$ that is not finitely presented. However, this is the only way in which homotopical and homological finiteness properties differ:

For $m \geq 2$, a group $G$ is of type $\mathrm{F}_{m}$ if and only if it is finitely presented and of type $\mathrm{FP}_{m}$.

Finiteness properties are still somewhat mysterious. Theorems relating finiteness properties in a transparent way to other, more group theoretic properties are in short supply. For special classes of groups, however, the situation is better. Eg, for metabelian groups, the Bieri-Strebel theory of geometric invariants 
leads to nice conjectures which are confirmed by a lot of examples and partial results. Finite presentability is well understood within this context. Eg, these groups are finitely presented if and only if they are of type $\mathrm{FP}_{2}$ [16].

In a situation like this, the best one can hope for is to get a feeling for the relationship between finiteness conditions and group structure within certain classes of groups. We will consider a class of $S$-arithmetic groups. These matrix groups are given by means of an algebraic group scheme $\mathcal{G}$ and a set $S$ of primes over a global field $K$ which determines an $S$-arithmetic ring $\mathcal{O}_{S} \subseteq K$. These two parameters can be varied independently, and one would like to know how finiteness properties vary with them. Moreover, these groups are natural generalizations of lattices in Lie groups, for which finiteness properties often have a more direct geometric interpretation. For all these reasons, a lot of research has already been done on finiteness properties of $S$-arithmetic groups.

The theory of $S$-arithmetic groups is dominated by two fundamental distinctions. The field $K$ can be a global number field or a global function field. With respect to the group scheme, there are two extremes the first of which is given by reductive groups, eg, $\mathrm{GL}_{n}$ or $\mathrm{SL}_{n}$, which is even a Chevalley group. Soluble groups, eg, groups of upper triangular matrices form the other extreme. Let us recall the most important results:

\section{$\mathcal{G}$ reductive:}

$K$ number field: $\mathcal{G}\left(\mathcal{O}_{S}\right)$ is of type $F_{\infty}$, ie, of type $\mathrm{F}_{m}$ for all $m \in \mathbb{N}$ [18.

$K$ function field: Finite generation and finite presentability are completely understood [9].

There are series of examples that support the conjecture that the finiteness length grows with $|S|$ and the rank of $\mathcal{G}$. The most important results are:

- $\mathrm{SL}_{2}\left(\mathcal{O}_{S}\right)$ is of type $\mathrm{F}_{|S|-1}$ but not of type $\mathrm{FP}_{|S|}$ [0].

- If $\mathcal{G}$ is a Chevalley group of rank $n$ not of exceptional type, then $\mathcal{G}(k[t])$ is of type $\mathrm{F}_{n-1}$ but not of type $\mathrm{FP}_{n}$ provided the finite field $k$ is large enough 6, Corollary 20, page 113].

\section{$\mathcal{B}$ soluble:}

$K$ number field: Finite generation and finite presentability are completely understood. Finite presentability is treated in [1].

In 41, Theorem 3.1], a Hasse principle is derived: $\mathcal{B}\left(\mathcal{O}_{S}\right)$ is of type $\mathrm{FP}_{m}$ if and only if for each place $p \in S$, the group $\mathcal{B}\left(\mathcal{O}_{p}\right)$ satisfies the compactness property $\mathrm{CP}_{m}$, which is defined in [3]. If $\mathcal{B}$ is 
a Borel subgroup of a Chevalley group, $\mathcal{B}\left(\mathcal{O}_{S}\right)$ is of type $\mathrm{F}_{\infty}$,41, Corollary 4.5].

Beyond these, there are some series of examples, eg, in [2].

$K$ function field: No Hasse principle holds in this case. This follows already from the series of metabelian groups that is examined using Bieri-Strebel theory in [24]. In this article, we generalize those results to group schemes of higher rank.

Our main result is the following:

Theorem A Let $\mathcal{G}$ be a Chevalley group, $\mathcal{B} \leq \mathcal{G}$ a Borel subgroup, $K$ a global function field, $S$ a non-empty set of places over $K$, and $\mathcal{O}_{S}$ the corresponding $S$-arithmetic ring. Then $\mathcal{B}\left(\mathcal{O}_{S}\right)$ is of type $\mathrm{F}_{|S|-1}$ but not of type $\mathrm{FP}_{|S|}$.

We will define notions and fix notations in the first two sections. Then we will deal with the rank-1-case. Sections 4 to 7 contain the proof of Theorem A: in Theorem 5.1 the upper bound is established whereas the lower bound is given in Theorem 7.5. The last section presents Theorem 8.5. which provides bounds for the "geometric invariants". Definitions and a bit of motivation will be given at the beginning of Section 8

This paper grew out of my $\mathrm{PhD}$ thesis, which I wrote under the supervision of Prof Robert Bieri. I would like to thank him for his support and encouragement. I also would like to thank the referee for very carefully reading the paper and suggesting numerous improvements.

\section{Preliminaries on adèles and unipotent groups}

General references about global fields and adèles are [27] or [45]. In this paper

- $K$ is a global function field. Its elements are called functions. Let

- $P$ denote the set of all places of $K$. We regard a place as a normalized discrete valuation $p: K \rightarrow \mathbb{Z} \cup\{\infty\}$. For each place $p$, there is a local function field

- $K_{p}$, which is the completion of $K$ at $p$. This is a topological field. Extending $p$ continuously, we obtain a normalized discrete valuation on $K_{p}$, which we also denote by $p$. The subring of functions holomorphic at $p$ is denoted by 
- $\mathcal{O}_{p}:=\left\{f \in K_{p} \mid p(f) \geq 0\right\}$. This ring is a compact open subspace of $K_{p}$. Moreover, it is a local ring with maximal ideal

- $\mathfrak{m}_{p}=\left\{f \in K_{p} \mid p(f)>0\right\}$. The constant functions, ie, the functions in $K$ that are holomorphic everywhere form a finite subfield

- $k$. The residue field

- $k_{p}:=\mathcal{O}_{p} / \mathfrak{m}_{p}$ is a finite extension of the field $k$ of degree

- $d_{p}:=\left[k_{p}: k\right]$. We define a norm on $K_{p}$ by

- $|f|_{p}:=e^{-d_{p} p(f)}$, which is proportional to the modulus of $K_{p}$.

Adèles provide a formalism to dealt with all places simultaneously. For a finite non-empty set $S \subseteq P$ of places,

- $\mathcal{O}_{S}:=\{f \in K \mid p(f) \geq 0 \quad \forall p \in P \backslash S\}$ is the ring of functions holomorphic outside $S$ and

- $\boldsymbol{A}_{S}:=\times_{p \in S} K_{p} \times \times_{p \notin S} \mathcal{O}_{p}$ is the topological ring of $S$-adèles.

The ring of adèles of $K$ is the direct limit

- $\boldsymbol{A}:=\lim _{S} \boldsymbol{A}_{S}$ of topological rings. For an adèle $\boldsymbol{a}=\left(f_{p}\right)_{p \in P} \in \boldsymbol{A}$, let

- $|\boldsymbol{a}|:=\prod_{p}\left|f_{p}\right|_{p}$ be the idèlic norm.

There is an inclusion $K \subseteq \boldsymbol{A}$ because every function in $K$ is holomorphic at almost all places. This way, $K$ is a discrete subring of $\boldsymbol{A}$, and we have

$$
\mathcal{O}_{S}=\boldsymbol{A}_{S} \cap K \text {. }
$$

Since we are working with topological rings, we regard a linear algebraic group $\mathcal{G}$ defined over a commutative ring $R$ as a functor from the category of (topological) commutative $R$-algebras into the category of (topological) groups.

A fundamental theorem says that the quotient $\boldsymbol{A} / K$ is compact 45, Theorem 2, page 64]. We need a slightly more general statement.

Lemma 1.1 Let $U$ be a unipotent linear algebraic group defined over $K$. Then $U(K)$ is a discrete subgroup of $U(\boldsymbol{A})$ and the quotients $U(\boldsymbol{A}) / U(K)$ and $U(K) \backslash U(\boldsymbol{A})$ are compact.

Proof Let $\widehat{U}$ denote the group of characters of $U$, ie, the linear algebraic group of $K$-morphisms from $U$ to $\mathfrak{M u l t}:=\mathrm{GL}_{1}$. Put

$$
U(\boldsymbol{A})^{\circ}:=\{g \in U(\boldsymbol{A})|| \chi(g) \mid=1 \text { for all } \chi \in \widehat{U}(K)\} .
$$

According to [17, Theorem 4.8], $U$ can be triagonalized over $K$. Hence, [8, Satz 3] implies that the quotient $U(\boldsymbol{A})^{\circ} / U(K)$ is compact. Therefore, it suffices to prove that $U(\boldsymbol{A})^{\circ}=U(\boldsymbol{A})$. This, however, follows from the fact that unipotent groups do not admit non-trivial characters, since homomorphisms of affine algebraic groups preserve unipotency [17, Theorem 4.4]. 


\section{Chevalley groups and the associated buildings}

We fix a Chevalley group

- $\mathcal{G}$, ie, a semisimple linear algebraic group defined over $\mathbb{Z}$. How to build such a group scheme is described, for example, in [28, [37, and [5]. A standard references for the following facts are [22] and [23].

Every Chevalley group comes with a root system

- $\Phi$. For any commutative unitary ring, we denote the group of $R$-points of $\mathcal{G}$ by

- $\mathcal{G}(R)$. If $K^{\prime}$ is a field, the group $\mathcal{G}\left(K^{\prime}\right)$ acts strong-transitively on the associated spherical building

- $\Delta\left(K^{\prime}\right)$ of type $\Phi$. Given a place $p$ on $K^{\prime}$, there is an affine building

- $X_{p}\left(K^{\prime}\right)$ associated to and acted upon by $\mathcal{G}\left(K^{\prime}\right)$. Affine buildings have apartments that are Euclidean Coxeter complexes. Using the Euclidean metric on apartments, the induced path-metric on an affine building is CAT(0). Moreover, apartments in affine buildings can be characterized in terms of this metric: a subspace is an apartment (in the complete system of apartments) if and only if it is a maximal flat subspace, ie, an isometrically embedded Euclidean space of maximal dimension. An excellent source and reference for the theory of affine buildings is 21, Chapter VI].

The building $\Delta\left(K^{\prime}\right)$ can be viewed in a natural way as the building at infinity of $X_{p}\left(K^{\prime}\right)$. If $K^{\prime}$ is complete with respect to $p$, then the system of apartments in $\Delta\left(K^{\prime}\right)$ induces the complete system of apartments in $X_{p}\left(K^{\prime}\right)$. Then we have a $1-1$-correspondence of spherical and affine apartments. In this case, we say that an affine apartment contains a chamber, a point, or a halfapartment at infinity if the corresponding spherical apartment does. Put

- $\Delta:=\Delta(K)$ for the fixed global function field $K$. Let

- $X_{p}:=X_{p}\left(K_{p}\right)$ denote the affine building associated to $\mathcal{G}\left(K_{p}\right)$ whereas

- $\Delta_{p}$ will denote the corresponding spherical building at infinity.

We fix a chain

$$
\mathcal{T} \leq \mathcal{B} \leq \mathcal{G}
$$

of group schemes defined over $\mathbb{Z}$ such that $\mathcal{T}(K)$ is a maximal $K$-split torus in $\mathcal{G}(K)$ and $\mathcal{B}(K)$ is a Borel subgroup, ie, a maximal solvable $K$-subgroup in $\mathcal{G}(K)$. Then, there is a unique apartment 
- $\Sigma_{p}$ in $X_{p}$ that is stabilized by $\mathcal{T}\left(K_{p}\right)$. We regard $\Sigma_{p}$ as the standard apartment. The group $\mathcal{T}\left(K_{p}\right)$ acts on $\Sigma_{p}$ as a maximum rank lattice of translations. Thus, the action of $\mathcal{T}\left(K_{p}\right)$ on $\Sigma_{p}$ is cocompact, ie, the quotient space of orbits of this action is compact.

Let

- $n$ be the dimension of $\mathcal{T}$. This is by definition the rank of $\mathcal{G}$. The building $X_{p}$ is a piecewise Euclidean complex of dimension $n$, and $\mathcal{G}\left(K_{p}\right)$ acts upon $X_{p}$ by cell-permuting isometries: every element of a cell stabilizer fixes the cell pointwise. Cell stabilizers are open and compact. Since $K_{p}$ is complete with respect to $p$, the group $\mathcal{G}\left(K_{p}\right)$ acts strongly-transitively on $X_{p}\left(K_{p}\right)$, ie, the action is transitive on the set of pairs $(\Sigma, c)$ where $\Sigma$ is an apartment in $X_{p}\left(K_{p}\right)$ and $c$ is a chamber in $\Sigma$.

The group $\mathcal{B}\left(K_{p}\right)$ is the stabilizer of a chamber at infinity

- $C_{p}$ in the standard apartment $\Sigma_{p}$. We call this the fundamental chamber at infinity. It is represented by a parallelity class of sectors in $X_{p}$. There is a canonical projection $\mathcal{B} \rightarrow \mathcal{T}$, which turns the torus $\mathcal{T}$ into a retract of $\mathcal{B}$. Let

- $\mathcal{U}$ denote its kernel, which is called the unipotent part of $\mathcal{B}$. The group $\mathcal{U}\left(K_{p}\right)$ not just stabilizes the fundamental chamber $C_{p}$, it fixes this chamber at infinity, ie, for each element in $\mathcal{U}\left(K_{p}\right)$, there is a sector representing $C_{p}$ which is fixed pointwise by the chosen element. This follows from the way the affine building $X_{p}$ and the action of $\mathcal{G}\left(K_{p}\right)$ on $X_{p}$ are constructed: the group $\mathcal{U}\left(K_{p}\right)$ turns out to be generated by root groups all of whose elements actually fix a Euclidean half apartment in $\Sigma_{p}$ containing $C_{p}$. The construction of the building is described in [22, Section 7.4] and the property of root groups used here is spelled out in Proposition (7.4.5) of said section. (These root groups are spherical and not to be confused with the affine root groups discussed in Section 6.)

Since any element of $\mathcal{U}\left(K_{p}\right)$ fixes a sector representing $C_{p}$, it cannot move chambers within the standard apartment $\Sigma_{p}$ at all: just consider a gallery in $\Sigma_{p}$ from a moved chamber to a chamber in the fixed sector. Since, on the other hand, $\mathcal{G}\left(K_{p}\right)$ acts strongly-transitively, $\mathcal{U}\left(K_{p}\right)$ acts on $X_{p}$ with $\Sigma_{p}$ as a fundamental domain. We thus obtain a projection map

- $\pi_{p}: X_{p} \rightarrow \Sigma_{p}$.

In order to determine the finiteness properties of $\mathcal{B}\left(\mathcal{O}_{S}\right)$, we will study its action on the product

- $\boldsymbol{X}:=\times_{p \in S} X_{p}$ of affine buildings. The projections $\pi_{p}$ induce a map

- $\boldsymbol{\pi}: \boldsymbol{X} \rightarrow \boldsymbol{\Sigma}:=\times_{p \in S} \Sigma_{p}$ onto the product of standard apartments. 
Lemma 2.1 The map $\boldsymbol{\pi}$ induces a proper map $\mathcal{U}\left(\mathcal{O}_{S}\right) \backslash \boldsymbol{X} \rightarrow \boldsymbol{\Sigma}$.

Proof Let $\boldsymbol{\sigma}:=\times_{p \in S} \sigma_{p}$ be a polysimplex in $\boldsymbol{X}$. For each place $p \in S$, the stabilizer $G_{p}$ of $\sigma_{p}$ in $\mathcal{U}\left(K_{p}\right)$ is an open compact subgroup. For $p \notin S$, we put $G_{p}:=\mathcal{U}\left(\mathcal{O}_{p}\right)$. Then $\boldsymbol{G}:=\times_{p} G_{p}$ is an open subgroup of $\mathcal{U}\left(\boldsymbol{A}_{S}\right)$.

There is an obvious action of $\mathcal{U}\left(\boldsymbol{A}_{S}\right)$ on $\boldsymbol{X}$ : Components outside $S$ act trivially whereas a component corresponding to a place $p \in S$ acts on the factor $X_{p}$. Hence $\boldsymbol{X}=\mathcal{U}\left(\boldsymbol{A}_{S}\right) \cdot \boldsymbol{\Sigma}$. The stabilizer of $\boldsymbol{\sigma}$ is $\boldsymbol{G}$ whence the fiber of $\boldsymbol{\pi}$ over $\boldsymbol{\sigma}$ is isomorphic to $\mathcal{U}\left(\boldsymbol{A}_{S}\right) / \boldsymbol{G}$ which in turn is a discrete set since $\boldsymbol{G}$ is open.

The group $\mathcal{U}\left(\mathcal{O}_{S}\right) \leq \mathcal{U}\left(\boldsymbol{A}_{S}\right)$ acts on $\mathcal{U}\left(\boldsymbol{A}_{S}\right) / \boldsymbol{G}$. Since $\mathcal{O}_{S}=K \cap \boldsymbol{A}_{S}$, Lemma 1.1 implies that the double quotient

$$
\mathcal{U}\left(\mathcal{O}_{S}\right) \backslash \mathcal{U}\left(\boldsymbol{A}_{S}\right) / G
$$

is discrete and compact. Thus, it is finite.

Therefore, the $\boldsymbol{\pi}$-fiber over each polysimplex consists of finitely many $\mathcal{U}\left(\mathcal{O}_{S}\right)-$ orbits of cells. Now the claim is evident.

Lemma 2.2 The group $\mathcal{G}\left(\mathcal{O}_{S}\right)$ acts on $\boldsymbol{X}$ with finite cell stabilizers.

Proof The cell stabilizers of the action of $\mathcal{G}\left(K_{p}\right)$ on $X_{p}$ are compact. Indeed, vertex stabilizers of this action are maximal compact subgroups of $\mathcal{G}\left(K_{p}\right)$ [22, Section 3.3]. Therefore, the stabilizer in $\mathcal{G}\left(\boldsymbol{A}_{S}\right)$ of a polysimplex in $\boldsymbol{X}$ is compact. The claim follows since $\mathcal{G}\left(\mathcal{O}_{S}\right)$ is a discrete subgroup of $\mathcal{G}\left(\boldsymbol{A}_{S}\right)$.

Each standard apartment $\Sigma_{p}$ is a Euclidean space of dimension $n$. Within each of them, we choose a sector

- $S_{p} \subseteq \Sigma_{p}$ representing the fundamental chamber $C_{p}$ of $\Delta_{p}$. We regard the cone point of $S_{p}$ as the origin in $\Sigma_{p}$ turning the apartment into a Euclidean vector space. Moreover, we represent all roots in $\Phi$ as linear forms on $\Sigma_{p}$. Following the usual convention, we call those of them negative that take negative values inside $S_{p}$. Thus we are given a system

- $\Phi_{p}^{-}$of negative roots in $\Sigma_{p}$. Considered as a subset of $\Phi$, it is independent of the place $p$ since all fundamental chambers $C_{p}$ correspond to the same Borel subgroup scheme $\mathcal{B}$. Passing to a set of base roots, we obtain a system of coordinates 
- $\varphi_{p}:=\left(\alpha_{1}^{(p)}, \ldots, \alpha_{n}^{(p)}\right)$ on $\Sigma_{p}$. With respect to these coordinates, the sector $S_{p}$ is given by

$$
S_{p}=\left\{x_{p} \in \Sigma_{p} \mid \alpha_{i}^{(p)}\left(x_{p}\right) \leq 0 \quad \forall i \in\{1, \ldots, n\}\right\} .
$$

Thus

$$
\boldsymbol{x}=\left(x_{p}\right)_{p \in S} \mapsto\left(\left(\alpha_{1}^{(p)}\left(x_{p}\right), \ldots, \alpha_{n}^{(p)}\left(x_{p}\right)\right)\right)_{p \in S}
$$

defines coordinates on $\boldsymbol{\Sigma}$. Scaling the different $p$-components appropriately, we arrange things such that the action of $\mathcal{T}\left(\mathcal{O}_{S}\right)$ leaves the map

- $\boldsymbol{\varphi}: \boldsymbol{x}=\left(x_{p}\right)_{p \in S} \mapsto\left(\sum_{p \in S} \alpha_{1}^{(p)}\left(x_{p}\right), \ldots, \sum_{p \in S} \alpha_{n}^{(p)}\left(x_{p}\right)\right)$ invariant. We can do so because the idelic norm is identically 1 on $K^{*}$ by the product formula [27, page 60]. The coordinates $\alpha_{i}^{(p)}$ correspond to base roots and, therefore, to characters $\chi_{i}: \mathcal{T} \rightarrow \mathfrak{M u l t}$. An element $\tau_{p} \in \mathcal{T}\left(K_{p}\right)$ acts on $\Sigma_{p}$ by a translation whose $i$-coordinate is $d_{p} p\left(\chi_{i}\left(\tau_{p}\right)\right)$.

We let $\mathcal{B}\left(\boldsymbol{A}_{S}\right)$ act on $\boldsymbol{\Sigma}$ via the projection $\mathcal{B} \rightarrow \mathcal{T}$. This way, $\boldsymbol{\pi}$ becomes a $\mathcal{B}\left(\boldsymbol{A}_{S}\right)$-map and hence a $\mathcal{B}\left(\mathcal{O}_{S}\right)$-map. Since this action of $\mathcal{B}\left(\mathcal{O}_{S}\right)$ on $\boldsymbol{\Sigma}$ factors through the torus $\mathcal{T}$ it leaves the map

- $\xi:=\varphi \circ \pi$ invariant.

Lemma 2.3 For any compact subset $\mathrm{C} \subseteq \mathbb{R}^{n}$, the preimage $\boldsymbol{\xi}^{-1}(\mathrm{C})$ contains a compact subset whose $\mathcal{B}\left(\mathcal{O}_{S}\right)$-translates cover $\boldsymbol{\xi}^{-1}(\mathrm{C})$.

Proof Dirichlet's Unit Theorem [27, page 72] implies that $\mathcal{T}\left(\mathcal{O}_{S}\right)$ acts cocompactly on the kernel of $\varphi$ whence it acts cocompactly on the preimage $\varphi^{-1}(\mathrm{C})$ as well. So, let $\mathrm{C}^{\prime} \subseteq \boldsymbol{\Sigma}$ be a compact set whose $\mathcal{T}\left(\mathcal{O}_{S}\right)$-translates cover $\varphi^{-1}(\mathrm{C})$.

By Lemma 2.1. we can find a compact subset $C^{\prime \prime} \subseteq \boldsymbol{X}$ whose $\mathcal{U}\left(\mathcal{O}_{S}\right)$-translates cover $\boldsymbol{\pi}^{-1}\left(\mathrm{C}^{\prime}\right)$. Then, the $\mathcal{B}\left(\mathcal{O}_{S}\right)$-translates of $\mathrm{C}^{\prime \prime}$ cover $\boldsymbol{\xi}^{-1}(\mathrm{C})$.

\section{Example: Rank-1-groups and trees}

It is as instructive as useful to treat the most simple case first: the Chevalley group $\mathrm{SL}_{2}$. Serre gives a comprehensive discussion of this group and its associated building in [36, II.1]. The group $D_{2}^{0}$ of diagonal matrices with determinant 1 is a maximal torus, and the group $B_{2}^{0}$ of upper triangular matrices with determinant 1 is a Borel subgroup. Its unipotent part is the group $U_{2}$ of strict upper triangular matrices all of whose diagonal entries equal 1. The affine building $X_{p}$ at the place $p$ is a regular tree of order $\left|k_{p}\right|+1$ : points in 
the link of a vertex correspond to points of the projective line over $k_{p}$. The standard apartment $\Sigma_{p}$ is a line. The projection map $\pi_{p}$ can be regarded as a height function on $X_{p}$ by identifying the apartment $\Sigma_{p}$ with the real line $\mathbb{R}$ via the negative base root $\alpha^{(p)}: \Sigma_{p} \rightarrow \mathbb{R}$. By scaling, as in the general case, we arrange that the action of $D_{2}^{0}\left(\mathcal{O}_{S}\right)$ on the product $\boldsymbol{X}:=\times_{p \in S} X_{p}$ leaves the height function $\boldsymbol{\xi}: \boldsymbol{X} \rightarrow \mathbb{R}$ invariant. This situation has been discussed already in [24]. Here we will treat it without making use of Bieri-Strebel theory.

Trees are crucial for everything that follows. Therefore, we will repeatedly make use of the following lemma, which may look somewhat technical at a first glance. However, it describes a rather natural geometrical situation.

Lemma 3.1 Let $\left(h_{i}: T_{i} \rightarrow \mathbb{R}\right)_{i \in\{1, \ldots, m\}}$ be a family of locally finite simplicial trees $T_{i}$ with height functions $h_{i}$. Suppose for every index $i$,

(1) $h_{i}$ maps the vertices of $T_{i}$ to a discrete subset of $\mathbb{R}$;

(2) there is exactly one descending end in $T_{i}$, ie, any two edge paths along which the height strictly decreases will eventually conincide; and

(3) each vertex in $T_{i}$ has degree $\geq 3$.

So all descending paths eventually meet, and every vertex has a unique lower neighbor and at least two higher neighbors.

Let $\boldsymbol{T}:=T_{1} \times \cdots \times T_{m}$ be the product of the trees $T_{i}$ and let $\boldsymbol{h}: \boldsymbol{T} \rightarrow \mathbb{R}$ be defined by

$$
\boldsymbol{h}: \boldsymbol{\tau}=\left(\tau_{1}, \ldots, \tau_{m}\right) \mapsto \sum_{i=1}^{m} h_{i}\left(\tau_{i}\right) .
$$

For every compact interval $I \subset \mathbb{R}$, put $\boldsymbol{T}[I]:=\boldsymbol{h}^{-1}(I)$.

Then, for each compact interval $I$, the space $\boldsymbol{T}[I]$ is $(m-2)$-connected, ie, the homotopy groups $\pi_{i}(\boldsymbol{T}[I])$ are trivial for $0 \leq i \leq m-2$.

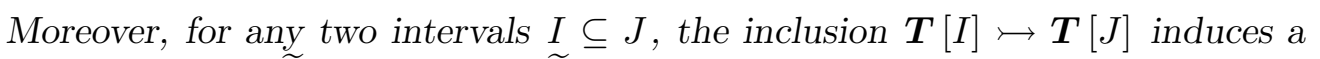
non-trivial map $\widetilde{\mathrm{H}}_{m-1}(\boldsymbol{T}[I]) \rightarrow \widetilde{\mathrm{H}}_{m-1}(\boldsymbol{T}[J])$ in reduced homology.

Proof The map $\boldsymbol{h}$ is a Morse function as defined in [11, Definition 2.2]. Its ascending and descending links in $\boldsymbol{T}$ are the joins of the ascending and descending links of the $h_{i}$ in the trees $T_{i}$, respectively. Thus, the descending links are points, and the ascending links are wedges of $(m-1)$-spheres. Hence ascending and descending links in $\boldsymbol{T}$ are $(m-2)$-connected. Then [11, Corollary 2.6] implies that $\boldsymbol{T}[I]$ is $(m-2)$-connected for each interval $I$ : The product $\boldsymbol{T}$ could not be contractible otherwise. 
As for the second claim, recall that each tree $T_{i}$ has a unique descending end. Moving every point in $T_{i}$ with unit speed downhill toward this end defines a flow on $T_{i}$. We obtain a flow on $\boldsymbol{T}$ that moves all points in $\boldsymbol{T}[I]$ toward $\boldsymbol{T}[\{\min (I)\}]$. This construction shows that $\boldsymbol{T}[\{\min (I)\}]$ is a strong deformation retract of $\boldsymbol{T}[I]$.

For $I \subseteq J$ the retraction $\boldsymbol{T}[J] \rightarrow \boldsymbol{T}[\{\min (J)\}]$ induces a map $\boldsymbol{T}[\{\min (I)\}] \rightarrow$ $\boldsymbol{T}[\{\min (J)\}]$ such that the following diagram

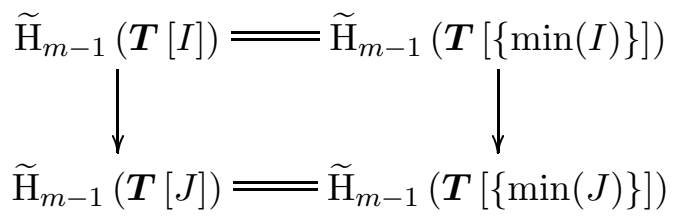

commutes. We will construct a sphere in $\boldsymbol{T}[\{\min (I)\}]$ which maps to a nontrivial embedded $(m-1)$-sphere in $\boldsymbol{T}[\{\min (J)\}]$. This proves the claim because the latter sphere defines a non-trivial cycle that cannot be a boundary because there is no $m$-skeleton in $\boldsymbol{T}[\{\min (J)\}]$.

We choose a point $\boldsymbol{\tau}=\left(\tau_{1}, \ldots, \tau_{m}\right) \in \boldsymbol{T}$ with $\boldsymbol{h}(\boldsymbol{\tau})<\min (J)$ all of whose coordinates $\tau_{i} \in T_{i}$ are vertices. For each $i$, we choose two ascending rays $L_{i}^{+}$ and $L_{i}^{-}$starting at $\tau_{i}$ without a common initial segment-recall that every vertex has at least two higher neighbors. The union $L_{i}:=L_{i}^{+} \cup L_{i}^{-}$is a line in $T_{i}$. The distance of a point $\tau^{\prime}{ }_{i} \in L_{i}$ to the "splitting vertex" $\tau_{i}$ is given by $h_{i}\left(\tau^{\prime}{ }_{i}\right)-h_{i}\left(\tau_{i}\right)$. Hence the map $\boldsymbol{\tau}^{\prime} \mapsto \boldsymbol{h}\left(\boldsymbol{\tau}^{\prime}\right)-\boldsymbol{h}(\boldsymbol{\tau})$ defines a norm on the product $\boldsymbol{L}:=\times_{i=1}^{m} L_{i}$.

The sphere we wanted is the sphere of all points in $\boldsymbol{L}$ whose norm is $\min (I)-$ $\boldsymbol{h}(\boldsymbol{\tau})$. The retraction shrinks it to the sphere of radius $\min (J)-\boldsymbol{h}(\boldsymbol{\tau})$, which is still strictly positive.

The rank-1-case is now easy since we can invoke KS Brown's celebrated criterion:

Citation 3.2 ([20, Remark (2) to Theorem 2.2 and Theorem 3.2]) Let $G$ be a group, $D$ a directed set, and $\left(X_{\alpha}\right)_{\alpha \in D}$ a directed system of $C W$-complexes on which $G$ acts by cell permuting homeomorphisms such that the following hold:

(1) For each $\alpha \in D$, the orbit space $G \backslash X_{\alpha}$ is compact.

(2) The stabilizer in $G$ of each $i$-cell in $X_{\alpha}$ is a group of type $F_{m-i}$.

(3) The continuous map $X_{\alpha} \rightarrow X_{\beta}$ indexed by $\alpha \leq \beta$ is $G$-equivariant. 
(4) The limit of the directed system of homotopy groups $\left(\pi_{i}\left(X_{\alpha}\right)\right)_{\alpha \in D}$ vanishes for $i<m$.

Then, $G$ is of type $F P_{m}$ if and only if for all $i<m$, the directed system of reduced homology groups $\left(\widetilde{\mathrm{H}}_{i}\left(X_{\alpha}\right)\right)_{\alpha \in D}$ is essentially trivial, ie, for each $\alpha \in D$, there is $\beta \geq \alpha$ such that the map $\widetilde{\mathrm{H}}_{i}\left(X_{\alpha}\right) \rightarrow \widetilde{\mathrm{H}}_{i}\left(X_{\beta}\right)$ induced by $X_{\alpha} \rightarrow X_{\beta}$ is trivial.

Moreover, $G$ is of type $F_{m}$ if and only if the directed system of homotopy groups $\left(\pi_{i}\left(X_{\alpha}\right)\right)_{\alpha \in D}$ is essentially trivial for all $i<m$.

Corollary 3.3 Let $G$ act cocompactly by cell-permuting homeomorphisms on an $(m-1)$-connected $C W$-complex $X$ such that the stabilizer of each cell is finite. Then $G$ is of type $F_{m}$.

Proof Take a directed set consisting of just one element, assign $X$ as the corresponding complex, and observe that the identity map induces trivial maps in homotopy groups in those dimensions where these groups vanish. Since finite groups are of type $\mathrm{F}_{\infty}$, the claim follows from Brown's Criterion.

Remark 3.4 In our applications, the direct limit of the spaces $X_{\alpha}$ will be the union of these spaces. Usually, it will be contractible which then implies that the limit of the directed system of homotopy groups $\left(\pi_{i}\left(X_{\alpha}\right)\right)_{\alpha \in D}$ vanishes for $i<m$.

Corollary 3.5 The group $B_{2}^{0}\left(\mathcal{O}_{S}\right)$ is of type $F_{|S|-1}$ but not of type $F P_{|S|}$.

Proof We apply Brown's Criterion. The set of all compact intervals in $\mathbb{R}$ is a directed set ordered by inclusion, and we are looking for a family of cocompact $B_{2}^{0}\left(\mathcal{O}_{S}\right)-\mathrm{CW}$-complexes over this system. $B_{2}^{0}\left(\mathcal{O}_{S}\right)$ acts on the product of trees $\boldsymbol{X}$ with $\boldsymbol{\xi}$ as an invariant height function. Hence for each compact interval $I$, the preimage $\boldsymbol{X}[I]:=\boldsymbol{\xi}^{-1}(I)$ is a $B_{2}^{0}\left(\mathcal{O}_{S}\right)$-complex. This defines our directed system with inclusions as continuous, $B_{2}^{0}\left(\mathcal{O}_{S}\right)$-equivariant maps.

The hypotheses of Brown's Criterion are satisfied: The action is by cellpermuting homeomorphisms, it is cocompact by Lemma 2.3. cell stabilizers are even finite by Lemma 2.2. and condition 4 is satisfied because the limit $\boldsymbol{X}$ of all $\boldsymbol{X}[I]$ is contractible.

The height function $\boldsymbol{\xi}$ can be regarded as a sum of height function defined on the factors $X_{p}$ such that we are in the setting of Lemma 3.1 the descending end in $X_{p}$ is the unique chamber at infinity (in this case just a point) stabilized by $B_{2}^{0}\left(K_{p}\right)$. This completes the proof. 
What can we say about the related group scheme $\mathrm{GL}_{2}$ ? The short exact sequence $\mathrm{SL}_{2} \longmapsto \mathrm{GL}_{2} \rightarrow \mathfrak{M u l t}$ with the determinant as the projection map induces by restriction a short exact sequence

$$
B_{2}^{0}\left(\mathcal{O}_{S}\right) \longrightarrow B_{2}\left(\mathcal{O}_{S}\right) \longrightarrow \mathcal{O}_{S}^{*}
$$

whence $B_{2}\left(\mathcal{O}_{S}\right)$ inherits all finiteness properties of $B_{2}^{0}\left(\mathcal{O}_{S}\right)$ since $\mathcal{O}_{S}^{*}$ is of type $\mathrm{F}_{\infty}$. However, $B_{2}\left(\mathcal{O}_{S}\right)$ might even exhibit stronger finiteness properties, but we can rule out this possibility:

Remark 3.6 The group $B_{2}\left(\mathcal{O}_{S}\right)$ is of type $\mathrm{F}_{|S|-1}$ but not of type $\mathrm{FP}_{|S|}$.

Proof Passing to projective groups, we obtain the following commutative diagram all of whose rows and columns are short exact sequences of groups:

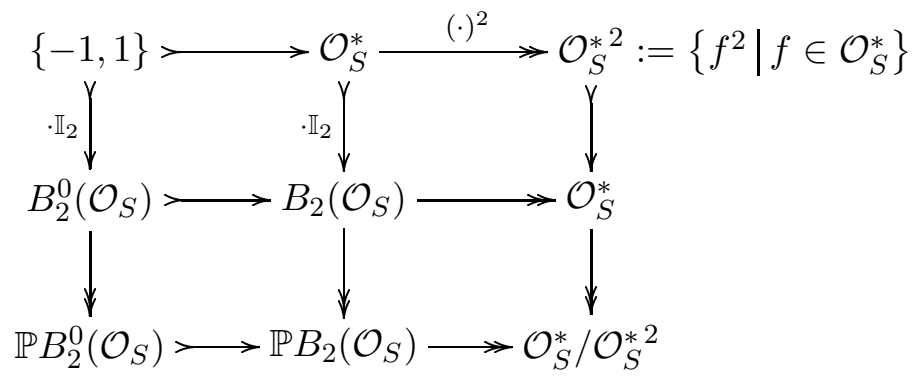

Consider the bottom row first. The factor $\mathcal{O}_{S}^{*} / \mathcal{O}_{S}^{* 2}$ on the right is an abelian torsion group which is finitely generated by Dirichlet's Unit Theorem. Hence $\mathcal{O}_{S}^{*} / \mathcal{O}_{S}^{* 2}$ is finite. Therefore, the other two groups in this row enjoy the same finiteness properties. Then, this also holds for their extensions in the middle row because the kernels on top are of type $\mathrm{F}_{\infty}$.

Remark 3.7 Of course, the same argument implies that $B_{n}^{0}\left(\mathcal{O}_{S}\right)$ and $B_{n}\left(\mathcal{O}_{S}\right)$ enjoy identical finiteness properties.

\section{Higher ranks - an algebraic prelude}

Perhaps the most striking consequence of Theorem A is that the finiteness length of $\mathcal{B}\left(\mathcal{O}_{S}\right)$ does not depend of the rank of the Chevalley group $\mathcal{G}$. In this section, a simple algebraic explanation for the group scheme $B_{n}^{0} \subseteq \mathrm{SL}_{n}$ is given. 
Proposition 4.1 Suppose $G \rightleftarrows H$ is a retract diagram of groups, ie, the composition of arrows is the identity on $H$. Then $H$ inherits all finiteness properties of $G$.

Proof Finite generation is trivial, finite presentability is easy and dealt with in [43. Lemma 1.3] where it is attributed to J R Stallings. Thus, it suffices to treat the homological finiteness properties starting with $\mathrm{FP}_{2}$. It would be possible to cite [4. ( $\zeta$ ), page 280], but Åberg is merely hinting at the argument. The following claims to be what he had in mind.

The key observation is, that functors and cofunctors both preserve retract diagrams. So for each index set $J$, consider the functor that assigns to a group $G$ the pair $\left(G, \times_{J} \mathbb{Z} G\right)$ where we regard $\times_{J} \mathbb{Z} G$ as a $\mathbb{Z} G$-module. Applying the homology functor $\mathrm{H}_{i}(-,-)$ yields the following retract diagram:

$$
\mathrm{H}_{i}\left(G, \times_{J} \mathbb{Z} G\right) \rightleftarrows \mathrm{H}_{i}\left(H, \times_{J} \mathbb{Z} H\right)
$$

Hence $\mathrm{H}_{i}\left(H, \times_{J} \mathbb{Z} H\right)$ vanishes whenever $\mathrm{H}_{i}\left(G, \times_{J} \mathbb{Z} G\right)$ does.

The claim now follows by means of the Bieri-Eckmann Criterion: A finitely generated group $H$ is of type $\mathrm{FP}_{m}$ if and only if $\mathrm{H}_{i}\left(H, \times_{J} \mathbb{Z} H\right)=0$ for all index sets $J$ and all $i \in\{1, \ldots, m-1\}$ [13, Proposition 1.2 and the equation above Theorem 2.3].

A different proof, based not on the Bieri-Eckmann Criterion but on Brown's Criterion, can be found in [26, Remark 3.3].

Corollary 4.2 The groups $B_{n}\left(\mathcal{O}_{S}\right)$ and $B_{n}^{0}\left(\mathcal{O}_{S}\right)$ are not of type $F P_{|S|}$.

Proof We confine ourselves to $n=3$. The group $B_{2}$ embeds into $B_{3}$ like this:

$$
B_{2} \cong\left(\begin{array}{ccc}
* & * & 0 \\
0 & * & 0 \\
0 & 0 & 1
\end{array}\right) \subseteq B_{3} .
$$

This way, we recognize $B_{2}$ as a retract of $B_{3}$. Hence, the preceding Proposition 4.1 applies and the claim follows from Corollary 3.5 and the Remarks 3.6 and 3.7 


\section{A geometric version of the argument}

Theorem 5.1 The group $\mathcal{B}\left(\mathcal{O}_{S}\right)$ is not of type $F P_{|S|}$.

The entire section is devoted to the proof of this theorem. The reasoning can be viewed as a geometric interpretation of the argument presented in the preceding section. Let us start with a brief outline: Each of the affine buildings $X_{p}$ contains a tree $T_{p}$ as a retract. Hence the product $\boldsymbol{X}$ contains a product $\boldsymbol{T}$ of trees as a retract. We will find a directed system of subspaces in $\boldsymbol{X}$ satisfying the hypotheses of Brown's Criterion. So we only have to prove that the induced system of reduced homology groups is not essentially trivial. Finally, using the retraction map, we pass to a corresponding system of subspaces in $\boldsymbol{T}$ where Lemma 3.1 applies.

Let us call an affine apartment in $X_{p}$ a layer if it contains the fundamental chamber at infinity $C_{p}$. The base root $\alpha^{(p)}:=\alpha_{1}^{(p)}$ defines half apartments in $X_{p}$ by

$$
\Sigma_{p}(t):=\left\{x_{p} \in \Sigma_{p} \mid \alpha^{(p)}\left(x_{p}\right) \leq t\right\} .
$$

Call an apartment in $X_{p}$ special if it contains such a half apartment. To put it in a slightly different way: The base root defines a half apartment at infinity $\alpha_{p}^{\infty} \subseteq \Delta_{p}$, which contains the fundamental chamber $C_{p}$. An affine apartment in $X_{p}$ is special if and only if it contains $\alpha_{p}^{\infty}$. Obviously, every special apartment is a layer.

The map $h_{p}:=\alpha^{(p)} \circ \pi_{p}$ restricts to an affine map on every layer-hence on every special apartment. Two special apartments $\Sigma_{p}^{1}$ and $\Sigma_{p}^{2}$ intersect in a convex set, which contains a subset of the form $\Sigma_{p}(s)$. Hence,

$$
\Sigma_{p}^{1} \cap \Sigma_{p}^{2}=\left\{x \in \Sigma_{p}^{1} \mid h_{p}(x) \leq t\right\}=\left\{x \in \Sigma_{p}^{2} \mid h_{p}(x) \leq t\right\}
$$

where $t=\max h_{p}\left(\Sigma_{p}^{1} \cap \Sigma_{p}^{2}\right)$. Thus we conclude:

Observation 5.2 The union of all special apartments in $X_{p}$ is a subcomplex isometric to a product $T_{p} \times \mathbb{R}^{n-1}$ where $T_{p}$ is a tree. The projection onto the second factor $\mathbb{R}^{n-1}$ is defined by $\left(\alpha_{2}^{(p)} \circ \pi_{p}, \ldots, \alpha_{n}^{(p)} \circ \pi_{p}\right)$. In particular, the fiber over each tuple $\left(t_{2}, \ldots, t_{n}\right)$ is a tree on which $h_{p}$ induces a height function $h_{p}^{*}: T_{p} \rightarrow \mathbb{R}$.

In [35. Chapter 10.2], $\mathrm{M}$ Ronan gives two constructions for the tree $T_{p}$. The equivalence of these two constructions underlies the following argument. 
Lemma 5.3 For each layer $\Sigma_{p}^{\prime}$, there is a special apartment $\Sigma_{p}^{\mathrm{s}}$ such that $h_{p}\left(\Sigma_{p}^{\prime} \cap \Sigma_{p}^{\mathrm{s}}\right)$ is unbounded.

Proof We argue within the spherical building $\Delta_{p}$. The apartment at infinity $\Sigma_{p}^{\prime \infty}$ corresponding to $\Sigma_{p}^{\prime}$ contains $C_{p}$. The root $\alpha_{p}^{\infty}$ determines a codimension 1 face of the fundamental chamber $C_{p}$. Let $C_{p}^{\prime}$ be the other neighboring chamber in $\Sigma_{p}^{\prime \infty}$. Then there is a unique apartment containing the chamber $C_{p}^{\prime}$ and the half apartment $\alpha_{p}^{\infty}$. The corresponding affine apartment $\Sigma_{p}^{\mathrm{s}}$ satisfies our needs since $h_{p}$ is unbounded on any sector representing $C_{p}^{\prime}$ in $\Sigma_{p}^{\mathrm{s}}$.

Lemma 5.4 Let $\Sigma_{p}^{1}$ and $\Sigma_{p}^{2}$ be special apartments and $\Sigma_{p}^{\prime}$ be a layer. Moreover, let $t$ be a real number and let $x_{p}^{i} \in \Sigma_{p}^{i} \cap \Sigma_{p}^{\prime}$ be two points such that $t=h_{p}\left(x_{p}^{i}\right)$. Then $t \in h_{p}\left(\Sigma_{p}^{1} \cap \Sigma_{p}^{2}\right)$.

Proof Both intersections $\Sigma_{p}^{i} \cap \Sigma_{p}^{\prime}$ are convex and contain a sector representing $C_{p}$. Let $S_{p}^{i}$ be the corresponding parallel sector with cone point $x_{p}^{i}$. Both sectors $S_{p}^{1}$ and $S_{p}^{2}$ are simplicial cones with a codimension 1 face restricted to which $h_{p}$ is identically $t$. Hence, these two faces intersect within $\Sigma_{p}^{\prime}$ and their intersection is a simplicial cone of codimension 1, which is contained in $\Sigma_{p}^{1} \cap \Sigma_{p}^{2}$

With the aid of the two lemmas above, we can see a projection $\rho_{p}: X_{p} \rightarrow T_{p}$.

Lemma 5.5 There is a continuous projection map $\rho_{p}: X_{p} \rightarrow T_{p}$ compatible with $h_{p}$, ie, the diagram

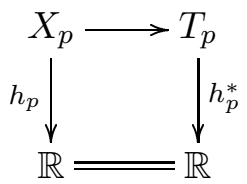

commutes where $h_{p}^{*}$ is as in Observation 5.2

Proof There is a 1-1-correspondence

$$
\left\{\text { special apartments in } X_{p}\right\} \leftrightarrow\left\{\begin{array}{l}
\text { lines in } T_{p} \text { that } h_{p}^{*} \\
\text { maps isometrically to } \mathbb{R}
\end{array}\right\}=: \mathcal{L} \text {. }
$$

According to Lemma 5.3. for each layer, there is a line onto which the layer can be projected in a way compatible with $h_{p}$. These projection maps agree where 
layers intersect by Lemma 5.4. Hence we have defined a projection map on $X_{p}$ since the affine building is the union of all layers.

This projection map is continuous when restricted to a layer since the tree $T_{p}$ carries the weak topology with respect to the lines in $\mathcal{L}$. Thus, the projection map is continuous because the building $X_{p}$ carries the weak topology with respect to the layers.

To see that this projection is a retraction, we need to find a continuous section. However, these exist in abundance by Observation 5.2

Consider the product

- $\boldsymbol{T}:=\times_{p \in S} T_{p}$, on which the height function

- $\boldsymbol{h}^{*}: \boldsymbol{T} \rightarrow \mathbb{R}$ is defined by

$$
\boldsymbol{\tau}=\left(\tau_{p}\right)_{p \in S} \mapsto \sum_{p \in S} h_{p}^{*}\left(\tau_{p}\right) .
$$

There is the projection map

- $\boldsymbol{\rho}: \boldsymbol{X} \rightarrow \boldsymbol{T}$ that admits a lot of sections, which are parameterized by tuples $\left(t_{p, i} \mid p \in S, i \in\{2, \ldots, n\}\right)$ of real numbers. Hence, $\boldsymbol{T}$ is a retract of $\boldsymbol{X}$

Observation 5.6 The following diagram commutes:

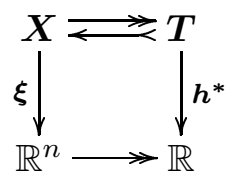

Here, the arrow in the bottom row is the projection onto the first coordinate.

Finally, we can turn to finiteness properties of $\mathcal{B}\left(\mathcal{O}_{S}\right)$. We want to apply Brown's Criterion; thus, we specify a directed system of cocompact $\mathcal{B}\left(\mathcal{O}_{S}\right)$ subcomplexes in $\boldsymbol{X}$ : A brick is a product $\boldsymbol{I}:=I_{1} \times \cdots \times I_{n} \subset \mathbb{R}^{n}$ of compact intervals. The set of bricks is a directed set ordered by inclusion. By Lemma 2.3, the family $\boldsymbol{X}[\boldsymbol{I}]:=\boldsymbol{\xi}^{-1}(\boldsymbol{I})$ of preimages of bricks is a directed system of cocompact $\mathcal{B}\left(\mathcal{O}_{S}\right)$-complexes.

Lemma 5.7 The system $\widetilde{\mathrm{H}}_{|S|-1}(\boldsymbol{X}[\boldsymbol{I}])$ of reduced homology groups is not essentially trivial. 
Proof Given a brick $\boldsymbol{I}$, let $\boldsymbol{J}:=J_{1} \times \cdots \times J_{n}$ be a brick containing $\boldsymbol{I}$, ie, $I_{i} \subseteq J_{i}$ for all $i$. Choose a tuple $\left(t_{p, i} \mid p \in S, i \in\{2, \ldots, n\}\right)$ of real numbers such that $\sum_{p \in S} t_{p, i} \in I_{i}$ for $2 \leq i \leq n$. This defines a section of $\boldsymbol{\rho}$, which restricts to a section

$$
\boldsymbol{T}\left[I_{1}\right]:=\boldsymbol{h}^{*-1}\left(I_{1}\right) \rightarrow \boldsymbol{X}\left[I_{1} \times\left\{\sum_{p \in S} t_{p, 2}\right\} \times \cdots \times\left\{\sum_{p \in S} t_{p, n}\right\}\right] \subseteq \boldsymbol{X}[\boldsymbol{I}] .
$$

Thus, Observation 5.6 implies that the preimage $\boldsymbol{T}\left[I_{1}\right]$ is a retract of $\boldsymbol{X}[\boldsymbol{I}]$ and that the diagram

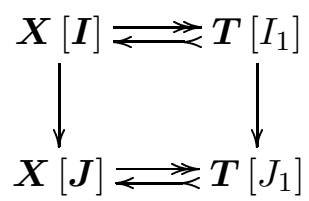

commutes. Passing to homology, we obtain the following commutative diagram:

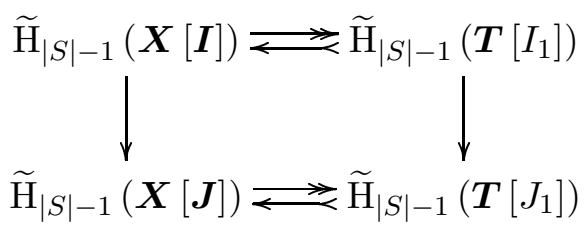

The right vertical arrow is non-trivial by Lemma 3.1 whence the left vertical arrow cannot be trivial either since the right hand side is a retract of the left hand side.

To finish the proof of Theorem [5.1, observe that the preimages $\boldsymbol{X}[\boldsymbol{I}]$ exhaust $\boldsymbol{X}$, which is contractible. The action of $\mathcal{B}\left(\mathcal{O}_{S}\right)$ on $\boldsymbol{X}$ is by cell-permuting homeomorphisms, and cell-stabilizers are finite by Lemma 2.2. Thus, in view of Brown's Criterion [3.2. Lemma 5.7 completes the proof of Theorem 5.1.

\section{The Moufang property}

Fix a building $X$ and an apartment $\Sigma$ therein. For any half apartment $\alpha$, let $-\alpha$ denote the complementary half apartment. Two half apartments $\alpha$ and $\beta$ in $\Sigma$ are prenilpotent if $\alpha \cap \beta$ contains a chamber and $-\alpha \cap-\beta$ contains a chamber, too. In this case, we set

- $[\alpha, \beta]:=\{\gamma \mid \alpha \cap \beta \subseteq \gamma,-\alpha \cap-\beta \subseteq-\gamma\}$ and

- $(\alpha, \beta):=[\alpha, \beta] \backslash\{\alpha, \beta\}$. 
$X$ is called Moufang if one can associate root groups

- $\mathrm{U}_{\alpha}$ of automorphisms of the building $X$ to the half apartments $\alpha \subseteq \Sigma$ such that the following axioms hold:

(M.1) $\mathrm{U}_{\alpha}$ fixes every chamber in $\alpha$, and for each panel $\pi \in \partial \alpha$ (a panel is a codimension 1 face of a chamber), $\mathrm{U}_{\alpha}$ acts simply-transitively on the set of chambers in $\operatorname{St}(\pi)$ but outside $\alpha$.

(M.2) For each prenilpotent pair $\{\alpha, \beta\}$, we have $\left[\mathrm{U}_{\alpha}, \mathrm{U}_{\beta}\right] \leq \mathrm{U}_{(\alpha, \beta)}$. Here, $\mathrm{U}_{(\alpha, \beta)}$ denotes the group generated by all $\mathrm{U}_{\gamma}$ with $\gamma \in(\alpha, \beta)$.

(M.3) For each $u \in \mathrm{U}_{\alpha} \backslash\{1\}$, there is an element $m(u) \in \mathrm{U}_{-\alpha} u \mathrm{U}_{-\alpha}$ stabilizing $\Sigma$.

(M.4) For $n=m(u)$ as in M.3, we have $n \mathrm{U}_{\beta} n^{-1}=\mathrm{U}_{n \beta}$ for all half apartments $\beta$.

Fact 6.1 The affine building associated to a Chevalley group $\mathcal{G}$ over a local function field is Moufang. In particular, this holds for the buildings $X_{p}$.

This is "well-known" to those who, well, know it. However, there seems to be no explicit reference for this fact in the literature. For this reason, an outline of the argument is included.

Sketch of proof By Hensel's Lemma or [45, Theorem 8, page 20], any local function field is isomorphic, as a field with a valuation, to a field of Laurent series over a finite field. This can be regarded as the completion of the field of rational functions over the same finite field. The associated buildings are isomorphic. Thus, we may confine ourselves to the case of a rational function field. As P Abramenko observes in [6, page 19], the corresponding building is isomorphic to the positive partner within the twin building of $\mathcal{G}$ over the ring of Laurent polynomials. He shows that this group has an RGD-system (confer $\underline{6}$, Definition 2, pages 14f]).

Finally one can derive the Moufang axioms from the RGD-axioms. This is not too difficult since the Moufang axioms M.2 to M.4 can be read as geometric interpretations of analogous RGD-axioms. The transitivity of the action in the Moufang axiom M.1 follows from [42, 5.6 Proposition 3, page 564] whereas (RGD 3) immediately implies that the action is simply-transitive.

In 4], $\mathrm{H}$ Åberg gave a method to detect the vanishing of homotopy groups for certain subspaces in products of trees. We will generalize his ideas to affine buildings. 
Definition 6.2 Let $X$ be an affine building and $C$ a chamber at infinity. We call a (not necessarily infinite) sequence of apartments $\left(\Sigma_{i}\right)$ directed if for each index $j$ the closed set

$$
\overline{\Sigma_{j} \backslash \bigcup_{i<j} \Sigma_{i}}
$$

is an intersection of half apartments in $\Sigma_{j}$ that do not contain $C$.

Proposition 6.3 If $X$ is locally finite and Moufang then there is an infinite directed sequence $\left(\Sigma_{i}\right)_{i \in \mathbb{N}}$ of apartments that covers $X$.

The proof will take the remainder of this section. So let us fix $X$ and $C$ as in the proposition. Further let us consider a bi-infinite geodesic gallery

- $g:=\left(\ldots, c_{i}, c_{i+1}, \ldots\right)$ within a chosen standard apartment

- $\Sigma$ that contains $C$. We will make more specific choices later. Let

- $\beta_{i}$ be the half apartment of $\Sigma$ containing $c_{i-1}$ but not $c_{i}$. We will write

- $\mathrm{U}_{i}:=\mathrm{U}_{\beta_{i}}$ to avoid double subscripts.

The following facts are quoted from [35, pages $74+]$ or can be proved by similar arguments without difficulty:

(1) $\mathrm{U}_{\alpha}$ fixes the star of every panel in $\alpha \backslash \partial \alpha$.

(2) $\mathrm{U}^{(r, s)}:=\left\langle\mathrm{U}_{i} \mid i \in\{r, \ldots, s\}\right\rangle=\mathrm{U}_{r} \cdots \mathrm{U}_{s}$. Moreover, the corresponding factorization $u=u_{r} \cdots u_{s}$ is unique for every element $u \in \mathrm{U}^{(r, s)}$.

(3) $\mathrm{U}_{r-1}$ and $\mathrm{U}_{s+1}$ normalize $\mathrm{U}^{(r, s)}$.

(4) $\mathrm{U}^{(r, s)}$ is finite since the building $X$ is locally finite.

Lemma 6.4 An element $u=u_{r} \cdots u_{s} \in \mathrm{U}^{(r, s)}$ fixes exactly those chambers of $\Sigma$ that lie in the intersection of all $\beta_{i}$ with $u_{i} \neq 1$. Moreover, this is the intersection $u \Sigma \cap \Sigma$.

Proof Put $u^{(i)}:=u_{r} \cdots u_{i}$. We proceed by induction on $i$. For $i=r$, we have $u^{(i)} \in \mathrm{U}_{r}$. If this element is trivial, then it fixes all of $\Sigma$, which we regard as the intersection of an empty family of half apartments. Now suppose $u_{r} \neq 1$ and assume that there was a chamber $c$ in $\Sigma \backslash \beta_{r}$ fixed by $u^{(r)}$. Then the element $u^{(r)}$ would fix every minimal gallery connecting $c$ to a chamber in $\beta_{r}$. In this case there would be a panel $\pi \in \partial \beta_{r}$ whose star contained two chambers fixed by $u^{(r)}$. This, however, is impossible by axiom M.1. 
Now suppose $i>r$. By induction, the lemma holds for $u^{(i-1)}$. Put $D:=$ $u^{(i-1)} \Sigma \cap \Sigma$. We have to show that for $u_{i} \neq 1$, the identity

$$
L:=D \cap \beta_{i}=u^{(i)} \Sigma \cap \Sigma=: R
$$

holds. By induction, we see that $c_{r-1} \in \beta_{r} \cap \cdots \cap \beta_{i} \subseteq L \subseteq R$ since $u_{i}$ fixes the half apartment $\beta_{i}$.

Assume there is a chamber $c \in R \backslash L$. Observe that $u^{(i)}$ stabilizes $c_{r-1}$ and preserves the type of minimal galleries. The chamber $c$ is uniquely determined by the type of a minimal gallery in $\Sigma$ connecting it to $c_{r-1}$. Hence $u^{(i)}$ fixes $c$ and also the gallery connecting $c$ to $c_{r-1}$. Consider the panel $\pi \in \partial L$ within this gallery. If $\pi \in \partial \beta_{i}$ then both of its neighboring chambers in $\Sigma$ lie in $D$ and $u_{i}$ fixes both of them which cannot happen. Therefore, $\pi$ lies in the interior of $\beta_{i}$. In this case, $u_{i}$ fixes the star of the panel $\pi$ whence $u^{(i-1)}$ has to fix both neighboring chambers in $\Sigma$. However, one of them does not belong to $D$ whence $u^{(i-1)}$ does not fix it. Thus, the hypothesis $L \varsubsetneqq R$ leads to a contradiction.

We will construct a sequence of automorphisms $\gamma_{j} \in\left\langle\mathrm{U}_{i} \mid i \in \mathbb{Z}\right\rangle \subseteq \operatorname{Aut}(X)$ that will give rise to a directed sequence $\Sigma_{j}:=\gamma_{j}(\Sigma)$. So we call a possibly finite sequence $\gamma_{1}, \ldots, \gamma_{i}, \ldots$ of automorphisms of $X$ directed if for each index $j$, the set

$$
\bigcup_{i<j} \gamma_{j}^{-1} \gamma_{i}(\Sigma) \cap \Sigma
$$

is coconvex, ie, the complement of a convex set. Here, we use the notion of convexity that stems from the Euclidean metric on $\Sigma$. However, recall that a subcomplex of the Coxeter complex $\Sigma$ is (metrically) convex if and only if it is an intersection of half apartments.

Observation 6.5 Suppose the gallery $g$ is chosen such that every half apartment $\beta_{i}$ contains the chamber $C$. Then every intersection $\gamma_{j}^{-1} \gamma_{i}(\Sigma) \cap \Sigma$ contains $C$. In this case, for any directed sequence $\left(\gamma_{i}\right)_{i \in \mathbb{N}}$ of automorphisms of $X$, the sequence $\left(\gamma_{i}(\Sigma)\right)_{i \in \mathbb{N}}$ is a directed sequence of apartments.

Lemma 6.6 Every directed enumeration of $\mathrm{U}^{(r, s)}$ can be extended to a directed enumeration of $\mathrm{U}^{(r-1, s+1)}$.

Proof We will only show that we can extend to $\mathrm{U}^{(r, s+1)}$. The argument for the lower index is similar. 
Let $\mathrm{U}^{(r, s)}=\left\{\gamma_{1}, \ldots, \gamma_{q}\right\}$ be a directed sequence enumerating $\mathrm{U}^{(r, s)}$ and choose an enumeration $\mathrm{U}_{s+1}=\left\{u_{1}, \ldots, u_{k}\right\}$ starting with $u_{1}=1$. Then

$$
\left\{u_{1} \gamma_{1}, \ldots, u_{1} \gamma_{q}, \ldots, u_{2} \gamma_{1}, \ldots, u_{2} \gamma_{q}, \ldots, u_{k} \gamma_{1}, \ldots, u_{k} \gamma_{q}\right\}
$$

is an enumeration of $\mathrm{U}^{(r, s+1)}$ which splits into $k$ blocks of length $q$. The first block is the given enumeration of $\mathrm{U}^{(r, s)}$. So we extended the enumeration. We will prove that this extension is directed. Within a block, quotients of elements agree with quotients in the first block. Thus, it suffices to prove that sets of the form

$$
\left(\bigcup_{\substack{\mu<\nu \\ i \leq q}} \gamma_{j}^{-1} u_{\nu}^{-1} u_{\mu} \gamma_{i}(\Sigma) \cap \Sigma\right) \cup\left(\bigcup_{i<j} \gamma_{j}^{-1} \gamma_{i}(\Sigma) \cap \Sigma\right)
$$

are coconvex.

The right hand term is coconvex because we extended a directed sequence.

If the left hand side is not empty it equals the half apartment $\beta_{s+1}$ : We have the factorization

$$
\gamma_{j}^{-1} u_{\nu}^{-1} u_{\mu} \gamma_{i}=\underbrace{\left(\gamma_{j}^{-1} u_{\nu}^{-1} u_{\mu} \gamma_{i} u_{\mu}^{-1} u_{\nu}\right)}_{\in \mathrm{U}^{(r, s)}} \underbrace{\left(u_{\nu}^{-1} u_{\mu}\right)}_{\in \mathrm{U}_{s+1}} .
$$

Since $u_{\nu}^{-1} u_{\mu} \neq 1$, Lemma 6.4 implies $\gamma_{j}^{-1} u_{\nu}^{-1} u_{\mu} \gamma_{i}(\Sigma) \cap \Sigma \subseteq \beta_{s+1}$. As $i \in$ $\{1, \ldots, q\}$ varies, the first factor $\gamma_{j}^{-1} u_{\nu}^{-1} u_{\mu} \gamma_{i} u_{\mu}^{-1} u_{\nu}$ runs through all of $\mathrm{U}^{(r, s)}$. In particular, at some point, $\gamma_{j}^{-1} u_{\nu}^{-1} u_{\mu} \gamma_{i} u_{\mu}^{-1} u_{\nu}$ will be the trivial element. Therefore, $\beta_{s+1}$ contains all sets $\gamma_{j}^{-1} u_{\nu}^{-1} u_{\mu} \gamma_{i}(\Sigma) \cap \Sigma$ and equals at least one of them.

Now, we choose the gallery $g$. We start with a straight line in the affine space $\Sigma$ that intersects each sector representing $C$. Note that any such line also intersects each sector representing the opposite chamber at infinity: geometrically such a line connects two antipodal points (in the spherical metric) of the spherical building at infinity. We move this line in its parallelity class so that it does not intersect the codimension 2 skeleton of $\Sigma$. We can do this, as the set of forbidden lines has measure 0 since the space of parallel lines has codimension 1. Given such a line, it intersects a sequence of chambers and thus defines the bi-infinite gallery $g=\left(\ldots, c_{i}, c_{i+1}, \ldots\right)$. By construction, all half apartments $\beta_{i}$ contain the chamber $C$, and the apartment $\Sigma$ is the convex hull of $g$, ie, there is no half apartment containing the whole gallery $g$.

By Lemma 6.6. we can find a directed sequence enumerating the group $\mathrm{U}^{(-\infty, \infty)}:=\left\langle\mathrm{U}_{i} \mid i \in \mathbb{Z}\right\rangle=\bigcup_{r, s, r \leq s} \mathrm{U}_{r} \cdots \mathrm{U}_{s}=\left\{\gamma_{i} \mid i \in \mathbb{N}\right\}$. By Observation 6.5 
this sequence induces a directed sequence $\Sigma_{i}:=\gamma_{i}(\Sigma)$ of apartments. To finish the proof of Proposition 6.3. it remains to show that these apartments cover $X$.

Let $\tilde{c}$ be a chamber in $X$. It suffices to show that there is an element $\gamma \in$ $\mathrm{U}^{(-\infty, \infty)}$ such that $\gamma \tilde{c} \subset \Sigma$. We will use induction on the length of a shortest gallery $\tilde{c}=c_{0}, c_{1}, \ldots, c_{r-1}, c_{r} \subset \Sigma$. Let $\alpha$ be the half apartment that contains $C$ and whose boundary contains the panel $c_{r-1} \cap c_{r}$. Since no half apartment contains the whole gallery $g$, we have $\mathrm{U}_{\alpha} \leq \mathrm{U}^{(-\infty, \infty)}$. By Axiom (M.1), there is an element $\gamma_{\alpha} \in \mathrm{U}_{\alpha}$ such that $\gamma_{\alpha} c_{r-1} \subset \Sigma$. Hence, $\gamma_{\alpha} \tilde{c}$ can be connected to $\Sigma$ by a shorter gallery than $\tilde{c}$. By induction, there is an element $\gamma^{\prime} \in \mathrm{U}^{(-\infty, \infty)}$ such that $\gamma^{\prime} \gamma_{\alpha} \tilde{c} \subset \Sigma$. This completes the proof of Proposition 6.3.

\section{Tameness, connectivity, and finiteness properties}

Let $X$ be a metric combinatorial $\mathrm{CW}$-complex such that the restriction of the metric to a closed cell makes the cell isometric to a convex Euclidean polyhedron, and let $\pi: X \rightarrow \mathbb{E}$ be a projection onto a Euclidean space $\mathbb{E}$. Furthermore, let us fix a non-empty set $\Psi$ of linear forms on $\mathbb{E}$. A sheet is a subcomplex of $X$ that $\pi$ maps isometrically onto $\mathbb{E}$. A closed subset of $\mathbb{E}$ is $\Psi$-convex if it is an intersection of halfspaces

$$
\bigcap_{\lambda \in \Psi^{\prime}}\left\{e \in \mathbb{E} \mid \lambda(e) \geq c_{\lambda}\right\}
$$

where $\Psi^{\prime}$ is a non-empty subset of $\Psi$ and $c_{\lambda} \in \mathbb{R}$ for every $\lambda \in \Psi^{\prime}$. In particular, $\mathbb{E}$ is not $\Psi$-convex unless $0 \in \Psi$. A closed subset of a sheet is called $\Psi$-convex if it is identified with a $\Psi$-convex subset of $\mathbb{E}$ via $\pi$. We follow the terminology of [24] and call the triple $(X, \mathbb{E}, \pi)$ a $\Psi$-complex if every compact subset of $X$ is covered by an increasing sequence of sheets. Here we call a sequence $\left(B_{i}\right)_{i}$ increasing if, at every stage, the piece $\overline{B_{s} \backslash \bigcup_{r<s} B_{r}}$ just added is $\Psi$-convex. Note that the set of sheets in a $\Psi$-complex $X$ form a cover of $X$.

Observation 7.1 Proposition 6.3 applies in particular to the building $X_{p}$ and the chamber $C_{p}$. Thus, $\left(X_{p}, \Sigma_{p}, \pi_{p}\right)$ is a $\Phi_{p}^{-}$-complex.

By [24, Example 7.2], we can lift this result to the product $\boldsymbol{\Sigma}$ :

Consequence 7.2 Each linear form in $\Phi_{p}^{-}$induces by composition with the projection $\boldsymbol{\Sigma} \rightarrow \Sigma_{p}$ a linear form on $\boldsymbol{\Sigma}$. In this sense, put 
- $\boldsymbol{\Phi}^{-}:=\bigcup_{p \in S} \Phi_{p}^{-}$.

Then $(\boldsymbol{X}, \boldsymbol{\Sigma}, \boldsymbol{\pi}: \boldsymbol{X} \rightarrow \boldsymbol{\Sigma})$ is a $\boldsymbol{\Phi}^{-}$-complex.

We will study the action of $\mathcal{B}\left(\mathcal{O}_{S}\right)$ on the complex

- $\boldsymbol{Y}:=\boldsymbol{\xi}^{-1}(0)=\boldsymbol{\pi}^{-1}(\boldsymbol{H})$ where

- $\boldsymbol{H}:=\operatorname{ker} \boldsymbol{\varphi}$. The map $\boldsymbol{\pi}: \boldsymbol{X} \rightarrow \boldsymbol{\Sigma}$ obviously restricts to $\boldsymbol{Y}$ and yields a projection $\boldsymbol{Y} \rightarrow \boldsymbol{H}$, which we will also denote by $\boldsymbol{\pi}$.

The linear forms in $\boldsymbol{\Phi}^{-}$restrict to $\boldsymbol{H}$. Let

- $\widetilde{\boldsymbol{\Phi}^{-}}:=\left.\boldsymbol{\Phi}^{-}\right|_{\boldsymbol{H}}$ be the system of linear forms obtained this way.

Observation 7.3 Let $(X, \mathbb{E}, \pi)$ be a $\Psi$-complex and $\mathbb{E}^{\prime}$ be a subspace of $\mathbb{E}$. Then $\left(\pi^{-1}\left(\mathbb{E}^{\prime}\right), \mathbb{E}^{\prime},\left.\pi\right|_{\mathbb{E}^{\prime}}\right)$ is a $\left.\Psi\right|_{\mathbb{E}^{\prime}}$-complex. Here, $\left.\Psi\right|_{\mathbb{E}^{\prime}}$ denotes the set of those linear forms in $\mathbb{E}^{\prime}$ obtained by restricting a linear form in $\Psi$ to $\mathbb{E}^{\prime}$. In particular, $(\boldsymbol{Y}, \boldsymbol{H}, \boldsymbol{\pi})$ is a $\widetilde{\boldsymbol{\Phi}^{-}}$-complex.

Note that the metric on $\pi^{-1}\left(\mathbb{E}^{\prime}\right)$ is not the path metric but the restriction of the metric on $X$. Moreover, in general, elements of $\Psi$ could vanish on $\mathbb{E}^{\prime}$. Observe, however, that $0 \notin \widetilde{\boldsymbol{\Phi}^{-}}$because of the product formula.

Lemma $7.4 \quad \boldsymbol{Y}$ is $(|S|-2)$-connected.

Proof By [24, Lemma 7.3] $\boldsymbol{Y}=\boldsymbol{\xi}^{-1}(0)=\boldsymbol{\pi}^{-1}(\boldsymbol{H})$ is $(|S|-2)$-connected provided $\widetilde{\boldsymbol{\Phi}^{-}}$is $(|S|-1)$-tame, ie, no positive linear combination of up to $|S|-1$ elements in $\widetilde{\boldsymbol{\Phi}^{-}}$vanishes.

Let us call a positive combination of forms in $\mathbf{\Phi}^{-}=\biguplus_{p \in S} \Phi_{p}^{-}$complete if it involves for each $p \in S$ at least one element of $\Phi_{p}^{-}$non-trivially. We shall argue that any positive combination of forms in $\boldsymbol{\Phi}^{-}$that vanishes on $\boldsymbol{H}$ is complete and hence involves at least $|S|$ forms with strictly positive weight. This implies that $\widetilde{\mathbf{\Phi}^{-}}=\left.\boldsymbol{\Phi}^{-}\right|_{\boldsymbol{H}}$ is $(|S|-1)$-tame.

Note that a positive combination of complete forms is complete. Since each element of $\Phi_{p}^{-}$is a positive combination of of base roots $\left(\alpha_{1}^{(p)}, \ldots, \alpha_{n}^{(p)}\right)$, we can write any positive combination of elements of $\boldsymbol{\Phi}^{-}$as a positive combination of base roots. Thus, it suffices to prove:

If $\sum_{i, p} \mu_{i, p} \alpha_{i}^{(p)}$ is a positive combination of base roots that vanishes on $\boldsymbol{H}$, then for each $p \in S$, there is an $i \in\{1, \ldots, n\}$ with $\mu_{i, p}>0$. 
This, however, follows from the definition of $\boldsymbol{H}$ since $\sum_{i, p} \mu_{i, p} \alpha_{i}^{(p)}$ vanishes on $\boldsymbol{H}$ only if, for each $i$, the coefficient $\mu_{i, p}$ does not depend on $p$.

We are now in a position to tackle the companion of Theorem 5.1

Theorem 7.5 The group $\mathcal{B}\left(\mathcal{O}_{S}\right)$ is of type $F_{|S|-1}$.

Proof $\mathcal{B}\left(\mathcal{O}_{S}\right)$ acts on $\boldsymbol{Y}$ by cell-permuting homeomorphisms. The action is cocompact by Lemma 2.3 The cell stabilizers are finite by Lemma 2.2 The space $\boldsymbol{Y}$ is $(|S|-2)$-connected by Lemma 7.4. Hence, the claim follows from Corollary 3.3 .

Remark 7.6 The proof of Theorem [7.5 mimics the argument given in [24] and uses the fact that the buildings $X_{p}$ are Moufang, which is a global property. Hence, this method does not apply to arithmetic groups over number fields as the associated buildings are not Moufang. However, the discussion in Section 3 indicates that it might be possible to find an argument for Theorem 7.5 somewhat in the spirit [1] that relies only on local properties of the buildings $X_{p}$.

Theorems [5.1] and 7.5 together imply Theorem A stated in the introduction.

\section{The geometric invariants of Bieri, Geoghegan, Neumann, Strebel and Renz}

"Geometric invariants", born in [16, are by now connected with five names. It would be unfeasible to draw a complete picture of the theory or its history. The reader may consult 34] or [15] where high dimensional invariants were first introduced. A more recent source is 12 .

The invariant $\Sigma^{m}(G)$ is defined for every group $G$ of type $\mathrm{F}_{m}$. It is an open cone in $\operatorname{Hom}(G, \mathbb{R})$. For each group $G$, we have a chain of inclusions

$$
\operatorname{Hom}(G, \mathbb{R}) \supseteq \Sigma^{1}(G) \supseteq \Sigma^{2}(G) \supseteq \cdots \supseteq \Sigma^{m}(G) .
$$

Definition 8.1 Let $G$ be a group of type $\mathrm{F}_{m}$ and $Y$ be an $(m-1)$-connected CW-complex upon which $G$ acts by cell permuting homeomorphisms such that the stabilizer of each $i$-cell is of type $\mathrm{F}_{m-i}$ and such that the action on the $m$-skeleton of $Y$ is cocompact. For each non-trivial homomorphism 
$\chi \in \operatorname{Hom}(G, \mathbb{R})$, there exists a $G$-equivariant height function on $Y$, ie, a continuous map $h_{\chi}: Y \rightarrow \mathbb{R}$ satisfying $h_{\chi}(g y)=\chi(g)+h_{\chi}(y)$ for all points $y \in Y$ and all elements $g \in G$.

Let $h_{\chi}$ be a height function associated to $\chi$. By definition $\chi \in \Sigma^{m}(G)$ holds if and only if $Y$ is essentially $(m-1)$-connected with respect to $h_{\chi}$, ie, the directed systems $\left(\pi_{i}\left(h_{\chi}^{-1}([t, \infty))\right)\right)_{t \in \mathbb{R}}$ of homotopy groups are essentially trivial for all $i<m$. For the definition of an essentially trivial directed system of groups, see Citation 3.2

Remark 8.2 One can show that this definition is independent of the complex $Y$ and the height function $h_{\chi}$ chosen to represent $\chi$. See [14, Theorem 12.1]. In earlier stages of the theory, the action of $G$ on $Y$ was required to be free. The weaker hypothesis regarding finiteness properties of cell stabilizers was proven to yield an equivalent definition of $\Sigma^{m}(G)$ by $\mathrm{H}$ Meinert in his $\mathrm{PhD}$ thesis. Its content is published in his papers [31] and 32. However, the reader might prefer the reference [14, Theorem 12.1] given above since that approach avoids a detour via homological invariants.

Remark 8.3 Still, our definition differs slightly from the literature. Since the difference lies in the order of quantifiers, this might be one of the few instances where logical formalism actually eases understanding. In the definition above, we require:

$$
\underset{i<m}{\forall} \underset{b \in \mathbb{R}}{\forall} \underset{a \leq b}{\exists} \pi_{i}\left(h_{\chi}^{-1}([b, \infty))\right) \rightarrow \pi_{i}\left(h_{\chi}^{-1}([a, \infty))\right) \text { vanishes. }
$$

Usually, the condition is phrased in a slightly stronger way:

$$
\underset{i<m}{\forall} \underset{l \geq 0}{\exists} \underset{b \in \mathbb{R}}{\forall} \pi_{i}\left(h_{\chi}^{-1}([b, \infty))\right) \rightarrow \pi_{i}\left(h_{\chi}^{-1}([b-l, \infty))\right) \text { vanishes. }
$$

However, since $G$ acts non-trivially on $\mathbb{R}$ by translations, both definitions are equivalent to the following even weaker condition:

$$
\underset{i<m}{\forall} \underset{b \in \mathbb{R}}{\exists} \underset{a \leq b}{\exists} \pi_{i}\left(h_{\chi}^{-1}([b, \infty))\right) \rightarrow \pi_{i}\left(h_{\chi}^{-1}([a, \infty))\right) \text { vanishes. }
$$

In particular, if $h_{\chi}^{-1}([b, \infty))$ is $(m-1)$-connected for some $b$, we have $\chi \in$ $\Sigma^{m}(G)$.

Generally, the geometric invariants of a group $G$ are hard to compute - they convey a lot of information. $\mathrm{Eg}, \Sigma^{m}(G)$ determines for each normal subgroup $N \unlhd G$ with abelian factor whether $N$ is of type $\mathrm{F}_{m}$ : 
Citation 8.4 (confer [34, Satz C, page 17]) Let $G$ be a group of type $F_{m}$ and $N \unlhd G$ be a normal subgroup with abelian factor group. Then, $N$ is of type $F_{m}$ if and only if all non-trivial homomorphisms in $\operatorname{Hom}(G, \mathbb{R})$ that vanish on $N$ belong to $\Sigma^{m}(G)$.

We already know that $\mathcal{B}\left(\mathcal{O}_{S}\right)$ is of type $\mathrm{F}_{|S|-1}$. Thus, the geometric invariants $\Sigma^{m}\left(\mathcal{B}\left(\mathcal{O}_{S}\right)\right)$ are defined for $1 \leq m<|S|$. Since we are working in positive characteristic, all unipotent groups are torsion groups. Thus, every homomorphism from $\mathcal{B}\left(\mathcal{O}_{S}\right)$ to $\mathbb{R}$ factors via $\mathcal{T}\left(\mathcal{O}_{S}\right)$ which spans a maximal lattice in the vector space $\boldsymbol{H}$ that can, therefore, be described as $\boldsymbol{H} \cong \mathcal{T}\left(\mathcal{O}_{S}\right) \otimes_{\mathbb{Z}} \mathbb{R}$. Thus,

- $\boldsymbol{H}^{*}:=\operatorname{Hom}_{\mathbb{R}}(\boldsymbol{H}, \mathbb{R}) \cong \operatorname{Hom}_{\mathbb{Z}}\left(\mathcal{T}\left(\mathcal{O}_{S}\right), \mathbb{R}\right) \cong \operatorname{Hom}\left(\mathcal{B}\left(\mathcal{O}_{S}\right), \mathbb{R}\right) \supseteq$ $\Sigma^{m}\left(\mathcal{B}\left(\mathcal{O}_{S}\right)\right)$. To avoid cumbersome notation, we will frequently use the complement

- $\Sigma^{m}\left(\mathcal{B}\left(\mathcal{O}_{S}\right)\right)^{\mathrm{c}}:=\boldsymbol{H}^{*} \backslash \Sigma^{m}\left(\mathcal{B}\left(\mathcal{O}_{S}\right)\right)$.

We will use the action of $\mathcal{B}\left(\mathcal{O}_{S}\right)$ on the space $\boldsymbol{Y}$ defined in Section 7 to compute the geometric invariants of $\mathcal{B}\left(\mathcal{O}_{S}\right)$. A homomorphism from $\mathcal{T}\left(\mathcal{O}_{S}\right)$ to the real numbers $\mathbb{R}$ can be represented by a linear form on $\boldsymbol{H}$. Composition with $\boldsymbol{\pi}: \boldsymbol{Y} \rightarrow \boldsymbol{H}$ yields a $\mathcal{B}\left(\mathcal{O}_{S}\right)$-equivariant height function on $\boldsymbol{Y}$.

We will derive a lower and an upper bound for $\Sigma^{m}\left(\mathcal{B}\left(\mathcal{O}_{S}\right)\right)^{c}$. These bounds will be sharp only in the rank-1-case. To state these bounds succinctly, we need some notation. Let

- $\boldsymbol{\Delta}^{-}:=\subseteq \boldsymbol{\Phi}^{-}$denote the set of all linear forms on $\boldsymbol{\Sigma}$ induced by base roots and let

- $\widetilde{\boldsymbol{\Delta}^{-}}:=\left.\boldsymbol{\Delta}^{-}\right|_{\boldsymbol{H}} \subseteq \widetilde{\boldsymbol{\Phi}^{-}}$be the restriction of $\boldsymbol{\Delta}^{-}$to $\boldsymbol{H}$. By

- $\operatorname{conv}_{m, S}\left(\widetilde{\boldsymbol{\Delta}^{-}}\right)$we refer to the set of all linear forms on $\boldsymbol{H}$ that can be written as a positive linear combination $\sum_{p \in S} \mu_{p} \alpha_{i_{p}}^{(p)}$ wherein at most $m$ coefficients do not vanish. Note that all base roots occurring in the combination belong to distinct places.

For an arbitrary set $\Psi$ of linear forms, let

- $\operatorname{conv}_{m}(\Psi)$ be the set of all linear forms that are positive combinations of up to $m$ elements of $\Psi$.

Theorem 8.5 For $m<|S|$, we have the chain of inclusions

$$
\operatorname{conv}_{m, S}\left(\widetilde{\boldsymbol{\Delta}^{-}}\right) \subseteq \Sigma^{m}\left(\mathcal{B}\left(\mathcal{O}_{S}\right)\right)^{\mathrm{c}} \subseteq \operatorname{conv}_{m}\left(\widetilde{\mathbf{\Phi}^{-}}\right) .
$$


Remark 8.6 In the rank-1-case, the upper and the lower bound coincide. Hence they determine $\Sigma^{m}\left(\mathcal{B}\left(\mathcal{O}_{S}\right)\right)^{\mathrm{c}}$. In this case, $\mathcal{B}\left(\mathcal{O}_{S}\right)$ is metabelian, and Theorem 8.5 establishes the $\Sigma^{m}$-conjecture for $\mathcal{B}\left(\mathcal{O}_{S}\right)$. This also follows from results of D Kochloukova [30].

Remark 8.7 It is easy to spell out some consequences of Theorem 8.5 in view of Citation 8.4 Let $A$ be a normal subgroup $\mathcal{B}\left(\mathcal{O}_{S}\right)$ containing $\mathcal{U}\left(\mathcal{O}_{S}\right)$. Such a subgroup always arises as a preimage of a subgroup $T^{\prime} \leq \mathcal{T}\left(\mathcal{O}_{S}\right)$. A homomorphism in $\operatorname{Hom}\left(\mathcal{B}\left(\mathcal{O}_{S}\right), \mathbb{R}\right)$ vanishes on $A$ if and only if the corresponding linear form in $\boldsymbol{H}^{*}$ vanishes on $\boldsymbol{H}_{T^{\prime}}:=T^{\prime} \otimes_{\mathbb{Z}} \mathbb{R} \leq \boldsymbol{H} \cong \mathcal{T}\left(\mathcal{O}_{S}\right) \otimes_{\mathbb{Z}} \mathbb{R}$. Thus, we are lead to consider $\boldsymbol{H}_{T^{\prime}}^{*}:=\left\{\lambda \in \boldsymbol{H}^{*} \mid \lambda\left(\boldsymbol{H}_{T^{\prime}}\right)=0\right\} \leq \boldsymbol{H}^{*}$. The codimension of $\boldsymbol{H}_{T^{\prime}}^{*}$ in $\boldsymbol{H}^{*}$ is nothing but the $\mathbb{Z}$-rank of $T^{\prime}$. According to Citation 8.4, $A$ is of type $\mathrm{F}_{m}$ provided the intersection $\Sigma^{m}\left(\mathcal{B}\left(\mathcal{O}_{S}\right)\right)^{\mathrm{c}} \cap \boldsymbol{H}_{T^{\prime}}^{*}$ is trivial.

By the upper bound of Theorem 8.5 $\Sigma^{m}\left(\mathcal{B}\left(\mathcal{O}_{S}\right)\right)^{\mathrm{c}}$ is a subset of $\operatorname{conv}_{m}\left(\widetilde{\mathbf{\Phi}^{-}}\right)$ which is a finite union of $m$-dimensional subspaces of $\boldsymbol{H}^{*}$. Hence $\Sigma^{m}\left(\mathcal{B}\left(\mathcal{O}_{S}\right)\right)^{c}$ has a trivial intersection with $\boldsymbol{H}_{T^{\prime}}^{*}$ provided the $\mathbb{Z}$-rank of $T^{\prime}$ is $\geq m$ and $\boldsymbol{H}_{T^{\prime}}$ (and hence $\boldsymbol{H}_{T^{\prime}}^{*}$ ) is "in general position".

The lower bound, analogously, implies that the $\mathbb{Z}$-rank of $T^{\prime}$ is at least $m$ if $A$ is of type $\mathrm{F}_{m}$. Hence in the "generic case", the finiteness length of $A$ is given by the minimum of $|S|-1$ and the $\mathbb{Z}$-rank of $T^{\prime}$.

Remark 8.8 On the other side, it is not difficult to find subgroups of $\mathcal{B}\left(\mathcal{O}_{S}\right)$ containing $\mathcal{U}\left(\mathcal{O}_{S}\right)$ that are not "in general position". Eg, consider the group

$$
\left\{\left(\begin{array}{ccc}
t & * & * \\
& t^{-2} & * \\
& & t
\end{array}\right) \in \operatorname{SL}_{3}\left(\mathcal{O}_{S}\right) \mid t \in \mathcal{O}_{S}^{*}\right\} .
$$

In the number field case, a related group is discussed in [2].

For $|S| \geq 2$, this group is finitely generated: we can write the elements in the upper right corner as products of commutators. This implies that the upper bound of Theorem 8.5 is not sharp.

However, independently of $|S|$, this group is not finitely presented: It is step2-nilpotent-by-abelian. If it was finitely presented then, by [16. Corollary 5.8], the subgroup in the upper right corner would be finitely generated as a normal subgroup. This is impossible since the torus acts trivially on it. It follows that the lower bound of Theorem 8.5 is not sharp, as well.

We now embark on the proof of Theorem 8.5] Both inclusions will be proved separately. The lower bound is derived in Lemma 8.9 and the upper bound in Corollary 8.12 
Lemma 8.9 For $m<|S|$, we have the inclusion:

$$
\operatorname{conv}_{m, S}\left(\widetilde{\boldsymbol{\Delta}^{-}}\right) \subseteq \Sigma^{m}\left(\mathcal{B}\left(\mathcal{O}_{S}\right)\right)^{\mathrm{c}}
$$

Proof Let $\lambda=\sum_{p \in S} \mu_{p} \alpha_{i_{p}}^{(p)}$ be a linear form on $\boldsymbol{H}$. Suppose $m$ coefficients are greater than 0 whereas the others vanish. We will apply the technique of Section 5 to prove that the system $\left(\pi_{m-1}\left(\left(\left.\lambda \circ \boldsymbol{\pi}\right|_{\boldsymbol{Y}}\right)^{-1}[t, \infty)\right)\right)_{t \in \mathbb{R}}$ is not essentially trivial.

In Section [5 we have seen how to construct a tree $T_{p}$ associated to a base root $\alpha_{i_{p}}^{(p)}$ that is a retract of the building $X_{p}$ and that carries a height function $h_{p}^{*}: T_{p} \rightarrow \mathbb{R}$ related to the height function $h_{p}=\alpha_{i_{p}}^{(p)} \circ \pi_{p}: X_{p} \rightarrow \mathbb{R}$ via the retraction map - see Lemma [5.5] and Observation [5.2

Put $\boldsymbol{T}^{\prime}:=\times_{\mu_{p} \neq 0} T_{p}$. The induced retraction $\times_{\mu_{p} \neq 0} X_{p} \rightarrow \boldsymbol{T}^{\prime}$ has a section $\sigma: \boldsymbol{T}^{\prime} \rightarrow \times_{\mu_{p} \neq 0} X_{p}$. Since $m<|S|$ there is at least one place $p \in S$ with $\mu_{p}=0$. Hence we find a continuous map $\rho: \boldsymbol{T}^{\prime} \rightarrow \times_{\mu_{p}=0} \Sigma_{p}$ such that, for each point $\boldsymbol{\tau}^{\prime} \in \boldsymbol{T}^{\prime}$, the tuple $\left(\rho\left(\boldsymbol{\tau}^{\prime}\right), \sigma\left(\boldsymbol{\tau}^{\prime}\right)\right) \in \times_{\mu_{p}=0} \Sigma_{p} \times \times_{\mu_{p} \neq 0} X_{p} \subseteq \boldsymbol{X}$ belongs to $\boldsymbol{Y}=\boldsymbol{\pi}^{-1}(\boldsymbol{H})$. Thus, we see that $\boldsymbol{T}^{\prime}$ is a retract of $\boldsymbol{Y}$.

Hence, $\sum_{p \in S} \mu_{p} h_{p}^{*}$ is a height function on $\boldsymbol{T}^{\prime}$ compatible with the height function induced by $\lambda$ on $\boldsymbol{Y}$, and the following diagram commutes:

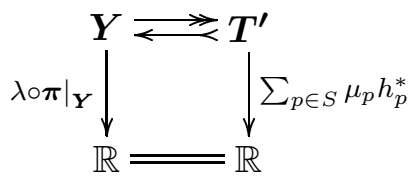

Thus, arguing as in the proof of Lemma 5.7 we see that the system $\left(\pi_{m-1}\left(\left(\left.\lambda \circ \boldsymbol{\pi}\right|_{\boldsymbol{Y}}\right)^{-1}[t, \infty)\right)\right)_{t \in \mathbb{R}}$ of homotopy groups is not essentially trivial since the corresponding system

$$
\left(\widetilde{\mathrm{H}}_{m-1}\left(\left\{\left(\tau_{p}\right) \in \boldsymbol{T}^{\prime} \mid t \leq \sum_{p \in S} \mu_{p} h_{p}^{*}\left(\tau_{p}\right)\right\}\right)\right)_{t \in \mathbb{R}}
$$

of reduced homology groups on the retract $\boldsymbol{T}^{\prime}$ is not essentially trivial by Lemma 3.1

We turn to the upper bound.

Observation 8.10 Let $\Psi$ be an $m$-tame set of linear forms on $\boldsymbol{H}$ and $\lambda$ be a linear form on $\boldsymbol{H}$. A positive combination of elements of $\Psi$ vanishes on ker $\lambda$ if and only if it belongs to the span of $\lambda$ in the dual $\boldsymbol{H}^{*}$. Hence, $\Psi$ restricts to an $m$-tame set of linear forms on ker $\lambda$ if and only if $\lambda \notin \operatorname{conv}_{m}(\Psi)$ and $-\lambda \notin \operatorname{conv}_{m}(\Psi)$. 
The following lemma, which is the key observation for the upper bound of Theorem [8.5] is a modification of [24, Lemma 7.3].

Lemma 8.11 Let $(X, \mathbb{E}, \pi)$ be a $\Psi$-complex and $\lambda$ be a linear form on $\mathbb{E}$. Suppose $\Psi$ is $m$-tame and $\lambda \notin \operatorname{conv}_{m}(\Psi)$. Then the preimage $X^{\prime}:=(\lambda \circ$ $\pi)^{-1}[0, \infty)$ is $(m-1)$-connected.

Proof Let $\left(B_{i}\right)_{i \in \mathbb{N}}$ be an increasing sequence in $X$. We will show that for each $r \in \mathbb{N}$, the space

$$
W^{r}:=\bigcup_{i \leq r} X^{\prime} \cap B_{i}
$$

is $(m-1)$-connected. Since any compact subset of $X$ is covered by some increasing sequence, it follows that $X^{\prime}$ is $(m-1)$-connected.

Observe that the case $r=1$ is trivial: $W^{1}=B_{1}$ is contractible. For $r>1$, assume by induction that $W^{r-1}$ is $(m-1)$-connected. We obtain $W^{r}$ from $W^{r-1}$ by gluing a closed convex set $V^{\prime} \subseteq B_{r}$ onto $W^{r-1}$ : Put $\mathbb{A}:=\lambda^{-1}[0, \infty)$ and $\mathbb{E}^{\prime}:=\operatorname{ker} \lambda=\partial \mathbb{A}$. We identify the sheet $B_{r}$ with $\mathbb{E}$ via the projection $\pi$. This way, $V^{\prime} \cong \mathbb{A} \cap V$ for a $\Psi$-convex set $V \subseteq \mathbb{E}$. The "new piece" $V^{\prime}$ is glued in along the set $\mathbb{A} \cap \partial V$. This process does not add material unless $\mathbb{E}^{\prime}$ contains an interior point of $V$ which we shall henceforth assume. In this case, $\mathbb{E}^{\prime} \cap \partial V=\partial(\mathbb{A} \cap V)$.

Using [24, Theorem 6.4] and its terminology, we distinguish two cases:

$V^{\prime}$ is decomposable: $V^{\prime}=V \cap \mathbb{A}$ is a product of a convex compact set and a Euclidean space, which is also a factor of $\mathbb{A}$. As we can split off this Euclidean factor, we may assume without loss of generality that $V^{\prime}$ is compact. Then, the intersection $V^{\prime} \cap \mathbb{E}^{\prime}$ is a "free face" of $V^{\prime}$, which we can push into-recall that $\mathbb{E}^{\prime}$ contains an interior point of $V$. Hence, $V^{\prime}$ can be collapsed onto $\partial V \cap \mathbb{A}$, which we thus recognize as a retract. Since this is the part along which $V^{\prime}$ is glued in, $W^{r}$ is homotopy equivalent to $W^{r-1}$.

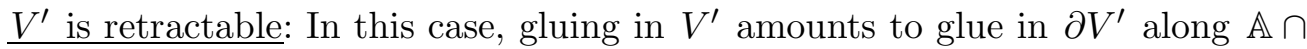
$\partial V$. However, we can retract everything toward $\mathbb{E}^{\prime}$. Thus, we could equivalently glue in $\partial V^{\prime} \cap \mathbb{E}^{\prime}=V \cap \mathbb{E}^{\prime}$ along $\partial V \cap \mathbb{E}^{\prime}=\partial\left(V \cap \mathbb{E}^{\prime}\right)$. So we have to determine the homotopy type of the pair $\left(V \cap \mathbb{E}^{\prime}, \partial\left(V \cap \mathbb{E}^{\prime}\right)\right)$.

Again, we have to distinguish two cases. Let $\Psi^{\prime} \subseteq \Psi$ be the set of all linear forms in $\Psi$ that correspond to hyperplanes supporting $V$. 
$-\lambda \notin \operatorname{conv}_{m}\left(\Psi^{\prime}\right)$ : We have seen in Observation 8.10 that in this case, the system $\Psi^{\prime}$ induces an $m$-tame system of linear forms on $\mathbb{E}^{\prime}$. Hence, $V \cap \mathbb{E}^{\prime}$ is either retractable or decomposable with a compact factor of dimension $\geq m$. The bound on the dimension follows from 24 , Corollary 6.7]. In either case, the gluing does not change homotopy groups in dimensions $<m$, ie, we have $\pi_{i}\left(W^{r}\right)=\pi_{i}\left(W^{r-1}\right)$ for $0 \leq i<m$.

$-\lambda \in \operatorname{conv}_{m}\left(\Psi^{\prime}\right):$ In this case, $\lambda$ is bounded from above on $V$. Since the set $V^{\prime}$ is retractable, it contains an infinite ray without its antipode. This ray cannot depart from $\mathbb{E}^{\prime}$ since otherwise $\lambda$ was unbounded on $V^{\prime} \subseteq V$. Hence the ray is parallel to $\mathbb{E}^{\prime}$. Thus, the set $V \cap \mathbb{E}^{\prime}$ is retractable as a subset of $\mathbb{E}^{\prime}$ : it contains a parallel ray. Hence gluing in this set does not change the homotopy type of $W^{r-1}$.

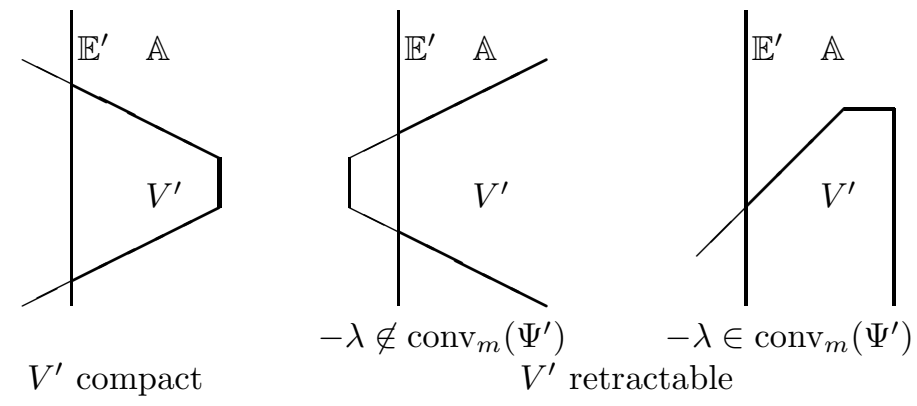

Corollary 8.12 For $m<|S|$, we have the inclusion $\Sigma^{m}\left(\mathcal{B}\left(\mathcal{O}_{S}\right)\right)^{\mathrm{c}} \subseteq$ $\operatorname{conv}_{m}\left(\widehat{\boldsymbol{\Phi}^{-}}\right)$.

Proof By Observation $\left[7.3,(\boldsymbol{Y}, \boldsymbol{H}, \boldsymbol{\pi})\right.$ is a $\widetilde{\boldsymbol{\Phi}^{-}}$-complex. In the proof of Lemma [7.4, we saw that $\widehat{\boldsymbol{\Phi}^{-}}$is $|S|$-tame. Thus the claim follows from Lemma 8.11 in view of Remark 8.3 .

Together with Lemma 8.9 this proves Theorem 8.5 


\section{References}

[1] H Abels, Finite presentability of S-arithmetic groups - Compact presentability of solvable groups, Springer LNM 1261 (1987)

[2] H Abels, K S Brown, Finiteness Properties of Solvable S-Arithmetic Groups: An Example, Journal of Pure and Applied Algebra 44 (1987) 77-83

[3] H Abels, A Tiemeier, Compactness Properties of Locally Compact Groups, Transformation Groups 2 (1997) 119-135 (see also 41])

[4] H Åberg, Bieri-Strebel Valuations (of Finite Rank), Proceedings of the London Mathematical Society 52 (1986) 269-304

[5] P Abramenko, Chevalleygruppen, Manuscript (Frankfurt, WS 1994/95)

[6] P Abramenko, Twin Buildings and Applications to S-Arithmetic Groups, Springer LNM 1641 (1996)

[7] G Baumslag, W W Boone, B H Neumann, Some Unsolvable Problems About Elements and Subgroups of Groups, Mathematica Scandinavica 7 (1959) 191-201

[8] $\mathbf{H}$ Behr, Endliche Erzeugbarkeit arithmetischer Gruppen über Funktionenkörpern, Inventiones Mathematicae 7 (1969) 1-32

[9] H Behr, Arithmetic groups over function fields; A complete characterization of finitely generated and finitely presented arithmetic subgroups of reductive algebraic groups, Preprint series 92-033, SFB 343; Bielefeld (1992); part of this is published in 10]

[10] H Behr, Arithmetic groups over function fields I; A complete characterization of finitely generated and finitely presented arithmetic subgroups of reductive algebraic groups, (Crelles) Journal für die reine und angewandte Mathematik 495 (1998) 79-118

[11] M Bestvina, N Brady, Morse theory and finiteness properties of groups, Inventiones mathematicae 129 (1997) 445-470

[12] R Bieri, Finiteness length and connectivity length for groups, from: "Geometric Group Theory down under", (J Cossey, CF Miller, W Neumann, M Shapiro, editors), Proceedings of the special year in geometric group theory, Australia National University, Canbera, 1996, deGruyter (1999) 9-22

[13] R Bieri, B Eckmann, Finiteness Properties of Duality Groups, Commentarii Mathematici Helvetici 49 (1974) 74-83

[14] R Bieri, R Geoghegan, Connectivity properties of group actions on nonpositively curved spaces, Memoirs of the American Mathematical Society 161 (2003) no. 765

[15] R Bieri, B Renz, Valuations on free resolutions and higher geometric invariants of groups, Commentarii Mathematicae Helvetici 63 (1988) 464-497 
Finiteness properties of soluble arithmetic groups

[16] R Bieri, R Strebel, Valuations and Finitely Presented Metabelian Groups, Proceedings of the London Mathematical Society 41 (1980) 439-464

[17] A Borel, Linear Algebraic Groups, Second Enlarged Edition Springer GTM 126, New York (1991)

[18] A Borel, J-P Serre, Cohomologie d'immeubles et de groupes S-arithmétiques, Topology 15 (1976) 211-232

[19] K S Brown, Cohomology of Groups, Springer GTM 87, New York (1982)

[20] K S Brown, Finiteness Properties of Groups, Journal of Pure and Applied Algebra 44 (1987) 45-75

[21] K S Brown, Buildings, Springer, New York, Berlin (1989)

[22] F Bruhat, J Tits, Groupes Réductives sur un Corps Local I, Publications Mathématiques IHES 41 (1972) 5-252

[23] F Bruhat, J Tits, Groupes Réductives sur un Corps Local II, Publications Mathématiques IHES 60 (1984) 5-184

[24] K-U Bux, Finiteness Properties of some Metabelian S-Arithmetic Groups, Proceedings of the London Mathematical Society 75 (1997) 308-322

[25] K-U Bux, Endlichkeitseigenschaften auflösbarer arithmetischer Gruppen über Funktionenkörpern, PhD thesis (Frankfurt, 1997)

[26] K-U Bux, Finiteness Properties Soluble S-Arithmetic Groups - A Survey, To appear: Conference Proceedings "Groups: Geometric and Combinatorial Aspects" Bielefeld 1999

[27] J W S Cassels, A Fröhlich, Algebraic Number Theory. Proceedings of an Instructional Conference organized by the London Mathematical Society (A Nato Advanced Study Institute) with Support of the International Mathematical Union, Academic Press, London (1967)

[28] C Chevalley, Certain Schémas de Groupes Semi-Simples, Séminaire Bourbaki 13e année (1960/61) no 219 1-16

[29] K W Gruenberg, J E Roseblade, Group Theory: Essays for Philip Hall, Academic Press (1984)

[30] D Kochloukova, The FP ${ }^{m}$-Conjecture for a class of metabelian groups, PhD thesis (Cambridge, 1997)

[31] H Meinert, The homological invariants for metabelian groups of finite Prüfer rank: a proof of the $\Sigma^{m}$-conjecture, Proceedings of the London Mathematical Society 72 (1996) 385-424

[32] H Meinert, Actions on 2-complexes and the homotopical invariant $\Sigma^{2}$ of a group, Journal of Pure and Applied Algebra 119 (1997) 297-317

[33] B H Neumann, Some remarks on infinite groups, Journal of the London Mathematical Society 12 (1937) 120-127 
[34] B Renz, Geometrische Invarianten und Endlichkeitseigenschaften von Gruppen, PhD thesis (Frankfurt, 1988)

[35] M Ronan, Lectures on Buildings, Perspectives in Mathematics, Academic Press, San Diego, London (1989)

[36] J-P Serre, Trees, Springer, Berlin Heidelberg New York (1980)

[37] R Steinberg, Lectures on Chevalley Groups, Mimeographed Notes, Yale University (1967)

[38] R Strebel, Finitely Presented Solvable Groups, from: 29] 257-314

[39] U Stuhler, Zur Frage der endlichen Präsentiertheit gewisser arithmetischer Gruppen im Funktionenkörperfall, Mathematische Annalen 224 (1976) 217-232

[40] U Stuhler, Homological properties of certain arithmetic groups in the function field case, Inventiones mathatematicae 57 (1980) 263-281

[41] A Tiemeier, A Local-Global Principle for Finiteness Properties of $S$ Artihmetic Groups over Number Fields, Transformation Groups 2 (1997) 215223 (see also [3])

[42] J Tits, Uniqueness and Presentations of Kac-Moody Groups over Fields, Journal of Algebra 105 (1987) 542-573

[43] C T C Wall, Finiteness Conditions for CW-Complexes, Annals of Mathematics 81 (1965) 56-69

[44] C T C Wall, Homological Group Theory, LMS Lecture Notes 35, Cambridge University Press (Cambridge 1979)

[45] A Weil, Basic Number Theory, Reprint of the Second Edition (1973) Springer Classics in Mathematics, Berlin Heidelberg (1995) 